\title{
A Differential-Exponential Hardening Law for Non-Schmid Crystal Plasticity Finite Element Modeling of Ferrite Single Crystals
}

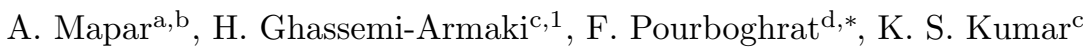 \\ ${ }^{a}$ Department of Mechanical Engineering, Michigan State University, MI 48824, USA \\ ${ }^{b}$ Department of Chemical Engineering and Materials Science, Michigan State University, \\ $M I$ 48824, USA \\ ${ }^{c}$ School of Engineering, Brown University, Providence, RI 02912 \\ ${ }^{d}$ Department of Integrated Systems Engineering, Department of Mechanical and Aerospace \\ Engineering, The Ohio State University, Columbus, OH 43210, USA
}

\begin{abstract}
Crystal plasticity finite element (CPFE) modeling of multi-phase, third generation advanced high strength steel (3GAHSS) requires finding the hardening parameters of slip systems operating in different phases (e.g. FCC, BCC, BCT). It is common to see the Schmid law used to model the deformation of BCC crystals. However, researches by Bassani and others have shown that BCC crystals could obey the non-Schmid law. In this paper we examined the differences between using a CPFE model based on the Schmid versus a non-Schmid law to model the uniaxial compression of single crystal ferrite micropillars with distinct orientations carved out of dual phase DP980 and three-phase QP980 steel sheets. To accurately model the changing hardening rate of the single crystal, a new exponential hardening model was developed that would differentially harden slip systems. Criteria for transitioning from stage I to stage II hardening of single crystals were also developed and verified.

Finally, it was shown that it is not sufficient to use only one single crystal micropillar compression force-displacement curve for the calibration of the non-Schmid CPFE model. The predictions of the resulting model would be
\end{abstract}

\footnotetext{
* Corresponding author

Email address: pourboghrat.2@osu.edu (F. Pourboghrat)

${ }^{1}$ Present address: ArcelorMittal Global R\&D, East Chicago, IN 46312
}

Preprint submitted to International Journal of Plasticity

November 4, 2016

(C) 2016. This manuscript version is made available under the Elsevier user license http://www.elsevier.com/open-access/userlicense/1.0/ 
unreliable, and its accuracy highly dependent on the orientation of the micropillar chosen for calibration. However, when two mircopillar compression force-displacement curves were used for calibration, the model's predictions were consistently accurate. Using more than two curves for calibration did not result in significant improvements.

Keywords: A. yield condition, B. crystal plasticity, C. finite elements, C. numerical algorithms, non-Schmid law

\section{Introduction}

Third generation advanced high strength steels (3GAHSS) are being developed for fabrication of lightweight automotive parts. These new steels have complex microstructures comprised of different phases, such as ferrite (BCC), 5 martensite (BCT), austenite (FCC), etc. with different volume fractions, grain sizes, and crystal orientations distributed throughout the material. Crystal plasticity finite element (CPFE) modeling of 3GAHSS is therefore becoming crucial for development of lightweight structures.

Accurate modeling of 3GAHSS using a CPFE model requires calibration of the hardening parameters of family of slip systems active in each phase (e.g. FCC and BCC). The calibration process involves repeated simulations of the uniaxial compression of a micropillar of a single phase (e.g. ferrite, martensite or austenite) with the CPFE model until there is a good match between predicted and experimentally measured force-displacement curves. The calibration could use just one or multiple experimental force-displacement curves. To assess the validity of the calibration, the calibrated CPFE model is used to simulate the uniaxial compression of the remaining micropillars that were not involved in the calibration process. In the case of $3 \mathrm{GAHSS}$, the CPFE model gets calibrated separately for each phase in the bulk material, as explained above. The collection of calibrated CPFE models for FCC and BCC phases will then be used to predict the behavior of the bulk material. The focus of this paper, however, is on modeling of the ferrite phase, which has a BCC crystal structure. 
Modeling the deformation of BCC materials is more challenging than FCC materials [1 5] due to the differences in the structure of dislocations. BCC materials have 48 slip systems but the slip planes are not ideally close-packed. The $1 / 2\langle 111\rangle$ screw dislocations in BCC metals have a non-planar core structure that spreads on three $\{1 \overline{1} 0\}$ planes. This causes the non-Schmid behavior, in which stresses developing on planes and directions other than those on the primary slip system will influence dislocation motion.

The non-planar structure of the core of screw dislocations in BCC metals has been known for decades [6]. Studies of the effect of stresses other than the glide stress on this non-planar core can be traced back to the work of Duesbery [7, 8. The first mechanistic model to capture the effect of the non-Schmid stresses, however, was proposed by Qin and Bassani [9, 10], which accounts for shear stresses affecting the core of screw dislocations. Terms for normal stresses affecting the core were later added to the model by Koester et al. [11. The non-Schmid behavior has been studied in many BCC materials including molybdenum [12 19], tungsten [15]22], tantalum [1], tantalum-tungsten alloy [23] and BCC iron [11, 24, 28].

40 This paper will be only concerned with the calibration and verification of the CPFE for modeling of single crystal BCC metals. The primary goal is to examine the differences between CPFE models based on Schmid and a non-Schmid law to predict the deformation of the ferrite phase in three-phase quenched and partitioned QP980 steels.

45 Another important issue to consider when calibrating the CPFE model is related to the stage I and stage II hardening of single crystals during the deformation [29]. There is very little hardening in stage I, due to the lack of competing slip systems and barriers to pin dislocations in a single crystal. However, in stage II rate of hardening increases, which is attributed to dislocation 50 entanglements and pile up caused by combination of the primary slip system rotating away from the loading direction, and secondary slip systems becoming activated with increasing deformation.

In this paper, we calibrated the CPFE model with single crystal ferrite mi- 
cropillars with different initial orientations. These micropillars were carved out of dual-phase DP980 and three-phase QP980 steels using Focused Ion Beam (FIB). The reason for using two different steels was twofold, a) deformation of ferrite in these two steels show non-Schmid behavior [25, 27, and b) the ferrite phase was compressed to about $4 \%$ strain in QP980 and only showed stage I hardening, while the strain reached double that amount in DP980 and showed both stage I and stage II hardening. Given that a conventional power-law hardening model could not represent the two-stage hardening of the ferrite phase, another important goal of this paper was to develop a novel phenomenological exponential hardening rule to be implemented into the CPFE model to predict the two-stage hardening of the ferrite phase. This new model, known as differential-exponential (DE) hardening, judiciously decides on the transitioning from stage I to stage II hardening when the following, a) the ratio of secondary to primary shear strain, and b) the ratio of the maximum shear stress to the saturation shear stress, are greater than the pre-specified values.

This paper is organized as follows. Section 2 reviews the basic formulation of crystal plasticity and explains the reasons behind the breakdown of the Schmid law in modeling the behavior of BCC materials. Section 3 describes the development of a rate-independent non-Schmid crystal plasticity model. Section 4 proposes a new hardening rule to improve the predictions of the non-Schmid crystal plasticity model in cases when the hardening rate changes during defor75 mation. This section also outlines the numerical implementation of the model for stress integration. Sections 5 and 6 discuss the predictions of the CPFE model for uniaxial compression of ferrite micropillars obtained from QP980 and DP980 steels, respectively. The conclusions drawn from this study are given in section 7. Finally, the Appendix A describes in detail the various strategies used for calibrating the hardening parameters and the non-Schmid material parameters. The comparison between experimental force-displacement curves and those predicted by the CPFE model are also presented. 


\section{Crystal plasticity model}

\subsection{Basic equations} defined with the Schmid tensor:

$$
P^{\alpha}=m^{\alpha} \otimes n^{\alpha}
$$

The velocity gradient tensor $L$ that is applied to a material point can be additively decomposed. tensors can be decomposed into elastic and plastic parts.

$$
D=D^{e}+D^{p}
$$

$$
W=\Omega+W^{p}
$$

where superscripts $e$ and $p$ denote elastic and plastic parts of the respective tensors. The elastic part of the spin tensor is the lattice spin rate tensor $\Omega$ 
130 135 140

150

155

160

which can be defined as follows:

$$
\Omega=R \dot{R}^{\top}
$$

125 where $R$ is the rigid body lattice rotation.

One can write the Schmid tensor $P^{\alpha}$ as the sum of a symmetric and an antisymmetric tensor:

$$
P^{\alpha}=d^{\alpha}+w^{\alpha}
$$

here $\alpha$ denotes the slip system, and $d^{\alpha}$ is the symmetric part of Schmid tensor defined as:

$$
d^{\alpha}=\frac{1}{2}\left(m^{\alpha} \otimes n^{\alpha}+n^{\alpha} \otimes m^{\alpha}\right)
$$

and $w^{\alpha}$ is the antisymmetric part of the Schmid tensor defined as:

$$
w^{\alpha}=\frac{1}{2}\left(m^{\alpha} \otimes n^{\alpha}-n^{\alpha} \otimes m^{\alpha}\right)
$$

Crystal plasticity assumes that all deformation is accommodated by dislocation slip. Therefore, the rate of plastic deformation $D^{p}$ needs to be accommodated by dislocation slip. This means that for a single crystal with $N$ slip systems $D^{p}$ is proportional to the shear $\dot{\gamma}^{\alpha}[10]$.

$$
D^{p}=\sum_{\alpha}^{N} d^{\alpha} \dot{\gamma}^{\alpha}
$$

The plastic spin rate is defined as:

$$
W^{p}=\sum_{\alpha}^{N} w^{\alpha} \dot{\gamma}^{\alpha}
$$

Therefore, one can find the lattice spin rate from:

$$
\Omega=W-\sum_{\alpha}^{N} w^{\alpha} \dot{\gamma}^{\alpha}
$$


The increment of rigid body rotation can be written in the following form [30]:

$$
R_{t_{0}+\Delta t}=R_{t_{0}}+\dot{R}_{t_{0}+\Delta t}=\left[I+R_{t_{0}} \cdot \dot{R}_{t_{0}+\Delta t} \Delta t\right] R_{t_{0}}=\left[I+\Omega_{t_{0}+\Delta t} \Delta t\right] R_{t_{0}}
$$

The grain orientation matrix $Q$ can be updated in a similar fashion with

$$
Q_{t_{0}+\Delta t}=Q_{t_{0}}+\Delta Q=\left[I+\Omega_{t_{0}+\Delta t} \Delta t\right] Q_{t_{0}}
$$

where $\Omega_{t_{0}+\Delta t}$ is the increment of lattice spin rate tensor in the finite element coordinate system. More details on updating crystal orientation can be found in 31 and 30 .

A general stress state applied on a crystal can induce a shear stress parallel to slip direction on the slip system $\alpha$. This shear stress can be found from the Schmid resolved shear stress equation:

$$
\tau^{\alpha}=\sigma: d^{\alpha}
$$

in which $\sigma$ is the stress tensor, and $\tau^{\alpha}$ is the resolved shear stress (RSS).

According to Schmid law, the dislocation motion starts when the in-plane shear stress parallel to the slip direction reaches a critical value. This is known as the critical resolved shear stress (CRSS). The CRSS is a material parameter and in FCC crystals is independent of the crystal orientation and the sense of loading (compression or tension).

\subsection{The non-Schmid model}

In BCC crystals, CRSS is a function of the orientation of the crystal with respect to the loading axis, the sense of loading [10], as well as stresses on planes that are non-parallel and/or non-planar with respect to the slip direction [11. These stresses are known as non-Schmid stresses. 
The yielding asymmetry of BCC single crystals in tension and compression has long been associated with twinning and anti-twinning sense of the shear 32. Recent studies, however, show this may be only partially true, as nonSchmid stresses have a significant impact on the yielding asymmetry [17. The non-Schmid stresses are also known as non-glide stresses because they affect the structure of the dislocation core and consequently the dislocation slip, but by themselves do not cause dislocation slip.

In BCC materials, the core of $1 / 2\langle 111\rangle$ screw dislocations spreads on three non-parallel $\{1 \overline{1} 0\}$ planes in the $\langle 111\rangle$ zone, which reduces their mobility, and in turn limits plastic flow [14. The core spreading is controlled by the crystal symmetry [14. This non-planar core leads to a violation of Schmid law and also accounts for the strain rate sensitivity and temperature dependence of the flow stress [11].

To capture the effect of non-glide stresses on the non-planar core and subsequently on the yielding of BCC materials, Qin and Bassani [9] proposed the following yield criterion which is a generalization of Schmid law.

$$
\tau^{\alpha}+\sum_{i=1}^{N_{n s}} a_{i}^{\alpha} \tau_{i}^{\alpha}=\tau_{c r}^{* \alpha}
$$

Here $\alpha$ corresponds to the slip system, $\tau_{c r}^{* \alpha}$ is the critical resolved shear stress value in the non-Schmid case, $\tau_{i}^{\alpha}$ and $N_{n s}$ are the non-Schmid stresses and their number respectively, $a_{i}^{\alpha}$ are material parameters, and $\tau^{\alpha}$ is the Schmid resolved shear stress. The expanded form of equation 15 is,

$$
\sum_{i=1}^{N_{n s}} a_{i}^{\alpha} \tau_{i}^{\alpha}=a_{1} \sigma:\left[m^{\alpha} \otimes n_{1}^{\alpha}\right]+a_{2} \sigma:\left[\left(n^{\alpha} \times m^{\alpha}\right) \otimes n^{\alpha}\right]+a_{3} \sigma:\left[\left(n_{1}^{\alpha} \times m^{\alpha}\right) \otimes n_{1}^{\alpha}\right]
$$

in which $n_{1}^{\alpha}$ is the plane normal of $\{1 \overline{1} 0\}$ in the slip direction zone, which also makes $-60^{\circ}$ with $n^{\alpha}$. The expanded non-Schmid yield criterion has the following 
$\tau_{c r}^{* \alpha}=\sigma:\left(m^{\alpha} \otimes n^{\alpha}\right)+a_{1} \sigma:\left(m^{\alpha} \otimes n_{1}^{\alpha}\right)+a_{2} \sigma:\left[\left(n^{\alpha} \times m^{\alpha}\right) \otimes n^{\alpha}\right]+a_{3} \sigma:\left[\left(n_{1}^{\alpha} \times m^{\alpha}\right) \otimes n_{1}^{\alpha}\right]$

Bassani et al. 12 combined all the geometric terms in a single parameter, $d^{* \alpha}$, that resembles the Schmid tensor in equation $[15$ as:

$$
\sigma: d^{* \alpha}=\tau_{c r}^{* \alpha}
$$

where

$$
d^{* \alpha}=d^{\alpha}+\sum_{\eta} a_{\eta}^{\alpha} d_{\eta}^{\alpha}
$$

in which $d_{\eta}^{\alpha}$ are the second rank tensors that represent the non-glide components

220

230 of the applied stress.

Although BCC materials yield according to a non-Schmid rule, they still deform by dislocation motion (slip) on slip planes. This means that the rate of plastic deformation $D^{p}$ is still proportional to the shear rate $\dot{\gamma}^{\alpha}[10$ as defined in the Schmid type case with equation (9).

225 In the Schmid type crystal plasticity model, $d^{\alpha}$ is used to define both the yield criterion and the flow potential [33]. This is known as the associated flow rule, and requires the increment of the plastic strain to be in the direction of the outward normal to the yield surface. A non-Schmid model, however, does not obey the associated plasticity law, and is known as the non-associated flow rule [34. In non-associated plasticity, two functions need to be defined, one to estimate stresses that cause yielding, and another to define the direction of the plastic strain.

The non-Schmid model has been verified for several BCC materials including BCC iron, molybdenum and tungsten, but there is still no consensus on the number of effective non-glide stress components. This number can vary for each material and may vary from one study to another. For instance, according 
to Gröger and Vitek [15], for tungsten the second term, and for molybdenum the third term, on the right hand side of equation (17) vanishes. To model BCC iron, Chen et al. 25] used all terms in equation (17) while Koester et al. [1] proposed a more general non-Schmid model with additional three non-glide normal stress terms included. This model is shown below:

$$
\begin{gathered}
\quad \sigma:\left[m^{\alpha} \otimes n^{\alpha}\right]+a_{1} \sigma:\left[m^{\alpha} \otimes n_{1}^{\alpha}\right]+a_{2} \sigma:\left[\left(n^{\alpha} \times m^{\alpha}\right) \otimes n^{\alpha}\right]+a_{3} \sigma:\left[\left(n_{1}^{\alpha} \times m^{\alpha}\right) \otimes n_{1}^{\alpha}\right] \\
+a_{4} \sigma:\left[n^{\alpha} \otimes n^{\alpha}\right]+a_{5} \sigma:\left[\left(n^{\alpha} \times m^{\alpha}\right) \otimes\left(n^{\alpha} \times m^{\alpha}\right)\right]+a_{6} \sigma:\left[m^{\alpha} \otimes m^{\alpha}\right]=\tau_{c r}^{* \alpha}
\end{gathered}
$$

The first term in this equation is the Schmid stress. The second to fourth terms incorporate the effect of shear stresses, and the fifth to seventh terms model the effect of normal stresses. Parameters $a_{1}$ to $a_{6}$ are material constants.

245 The planes that the resolved stresses, modeled with equation 20), act on are schematically shown in Figure 1. The red, green and blue planes are three $\{1 \overline{1} 0\}$. The red plane is the slip plane. The gold and purple planes are drawn only to facilitate the illustration of non-Schmid normal stresses. The gray line is the slip direction and is along $\langle 111\rangle$. The Schmid resolved shear stress is shown with a solid black line and is marked respectively. The non-Schmid resolved shear stresses are shown with dashed lines, and normal stresses are shown with solid lines and are marked with their respective non-Schmid constants $a_{1}-a_{6}$.

The term including $a_{1}$ is the shear stress parallel to the slip direction on any $\{1 \overline{1} 0\}$ plane other than the slip plane (the blue and green planes in Figure 11). 255 According to Gröger et al. [17, this stress causes the twinning-anti twinning effect. The term including $a_{2}$ is the shear stress normal to the slip direction acting in the slip plane; $a_{3}$ is the shear stress normal to slip direction acting on a $\{1 \overline{1} 0\}$ plane other than the slip plane (red plane in Figure 1); $a_{4}$ is the normal stress perpendicular to slip plane (and therefore perpendicular to the slip direction) acting on the slip plane; $a_{5}$ is the normal stress perpendicular to slip direction acting on a plane perpendicular to the slip plane (the gold plane in Figure 1); and finally $a_{6}$ is the normal stress parallel to slip direction acting on a 


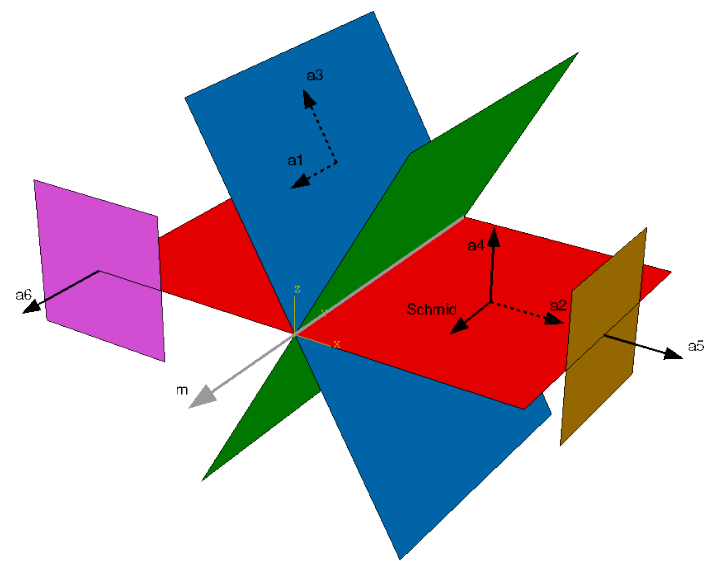

Figure 1: The non-Schmid planes and stresses that are considered in equation 20 are schematically shown here. The red, blue and green planes are the $\{1 \overline{1} 0\}$ planes. Here the red plane is the slip plane. The slip direction is shown with a gray line along $\langle 111\rangle$ direction. The Schmid resolved shear stress along the slip direction is shown with a solid black line on the red plane and in the slip direction. The non-Schmid resolved shear stresses are shown with dashed lines. For brevity the resolved shear stresses $a_{1}$ and $a_{3}$ are only shown on the blue plane, however, similar shear stresses may be resolved on the green plane. The gold and purple planes are not crystal planes and are only drawn to help with the visualization of the non-Schmid normal stresses. These stresses $\left(a_{4}, a_{5}\right.$ and $\left.a_{6}\right)$ are drawn with solid lines and act normal to the red, gold and purple planes.

plane perpendicular to slip direction (the purple plane in Figure 1). According to Koester et al. [11, the last term makes the yield criterion independent of the hydrostatic stress. Many researchers [11, 12, 14, 19, 34] have used atomistic simulation to find the material constants used in the non-Schmid models.

\section{Developing the non-Schmid, non-associated crystal plasticity model}

Crystal plasticity theory assumes that plastic deformation occurs as a result of dislocation motion on a slip plane. The current non-Schmid crystal plasticity model is developed following a Schmid type crystal plasticity framework that Zamiri and Pourboghrat proposed [33. The basic equations of this model are given in section 2.1. Zamiri and Pourboghrat formulated the yield function for 
a single crystal with multiple slip systems as:

$$
f_{\alpha}(\sigma, u)=\frac{\left|\sigma: d^{\alpha}\right|}{\tau_{y}^{\alpha}}-1 \leq 0 \quad \text { for } \quad \alpha=1 \ldots N
$$

where $u$ represents the internal variables, $\alpha$ is the slip system, $N$ is the total

275 is the critical resolved shear stress for slip system $\alpha$.

The yield surface defined with this equation is not smooth and may have sharp corners. Therefore, the outward normal to the yield surface at these points will not be unique and that implies that the plastic strain increment cannot be

uniquely identified. To address this problem, Zamiri and Pourboghrat [33] used the following scheme.

$$
G(\sigma, u)=\frac{1}{\rho} \ln \left\{\sum_{\alpha=1}^{N} \exp \left[\frac{\rho}{\mu}\left(\frac{\left|\sigma: d^{\alpha}\right|}{\tau_{y}^{\alpha}}-1\right)\right]\right\}
$$

This equation defines a smooth envelope inside the yield surface defined by equation 21). The parameter $\rho$ is termed the closeness factor and has an inverse relation with the distance between the smooth inner envelope and the original 285 yield surface. Parameter $\mu$ is used to further tune the yield function.

Assuming associated flow rule, the rate of the plastic deformation can be found from:

$$
D^{p}=\lambda \frac{\partial G(\sigma, u)}{\partial \sigma}
$$

where $\lambda$ is the plastic consistency parameter.

For BCC materials, as discussed above, the Schmid law does not apply. This 290 is one of the reasons why the crystal plasticity scheme that works well for FCC single crystals, such as aluminum, does not perform as well for BCC materials like $\mathrm{Nb}$ or BCC Iron.

Zamiri and Pourboghrat's combined constrained yield function is developed using the associated flow rule [33. This rule mandates the plastic strain increment to be in the direction of the outward normal to the yield surface. While this is the case for FCC materials, it is not true for BCC materials. 
To address this problem, and based on the above discussions, the authors developed a combined constrained non-associated (non-Schmid) crystal plasticity model by defining a yield function and an independent flow potential. effect of the glide and non-glide resolved stresses, $\tau_{y}^{*}=\sigma: d^{*}$, reaches a critical value, $\tau_{c r}^{*}$. For one slip system, the following function is used to indicate the onset of yielding.

$$
\frac{\sigma: d^{*}}{\tau_{c r}^{*}}=1
$$

One can arrive at the above equation simply by rearranging equation (17).

$$
\frac{\sigma: d}{\tau_{c r}^{*}}=1
$$

Notice that the numerator in equation 24 is the non-Schmid resolved stress, and in equation 25 is the Schmid resolved shear stress.

Following Zamiri and Pourboghrat, a smooth flow potential is defined based on equation 25 as:

$$
F(\sigma, u)=\frac{1}{\rho_{1}} \ln \left\{\sum_{\alpha=1}^{N} \exp \left[\frac{\rho_{1}}{\mu_{1}}\left(\frac{\left|\sigma: d^{\alpha}\right|}{\tau_{y}^{* \alpha}}-1\right)\right]\right\}
$$

where $\rho_{1}$ is the closeness factor and $\mu_{1}$ is a parameter to tune the flow potential. Then, the rate of plastic strain is defined as:

$$
D^{p}=\lambda \frac{\partial F(\sigma, u)}{\partial \sigma}
$$

And the rate of slip (shear rate) as:

$$
\dot{\gamma}^{\alpha}=\lambda \frac{\partial F(\sigma, u)}{\partial \tau^{\alpha}}
$$


Finally, the rate of increase in the critical resolved shear stress (yield stress) is modeled with the hardening model by Hill [35].

$$
\dot{\tau}_{y}^{\alpha}=\sum_{\beta=1}^{N} h^{\alpha \beta}\left|\dot{\gamma}^{\beta}\right|
$$

The hardening moduli matrix, $h^{\alpha \beta}$, is defined as follows [36]:

$$
h^{\alpha \beta}=h^{\beta}\left[q+(1-q) \delta^{\alpha \beta}\right]
$$

where $\delta^{\alpha \beta}$ is the Kronecker delta, and $q$ is the latent-hardening ratio which is defined as the ratio of latent-hardening to self-hardening rate and has values in and off-diagonal components are latent-hardening. Parameter $h^{\beta}$ is a function that defines the self-hardening rate and is defined using the following equation.

$$
h^{\beta}=h_{0}\left|1-\frac{\tau_{c}^{\beta}}{\tau_{s}}\right|^{\eta} \operatorname{sgn}\left(1-\frac{\tau_{c}^{\beta}}{\tau_{s}}\right)
$$

Here, $h_{0}$ is the initial hardening rate, $\tau_{c}^{\beta}$ is the current slip resistance on slip system $\beta, \tau_{s}$ is the saturation value of slip resistance, and $\eta$ is the exponent controlling the hardening rate.

Equation (24) is then used to define the following smooth non-Schmid yield function,

$$
Y(\sigma, u)=\frac{1}{\rho_{2}} \ln \left\{\sum_{\alpha=1}^{N} \exp \left[\frac{\rho_{2}}{\mu_{2}}\left(\frac{\left|\sigma: d^{* \alpha}\right|}{\tau_{y}^{* \alpha}}-1\right)\right]\right\}
$$
where $\rho_{2}$ is the closeness factor and $\mu_{2}$ is a parameter to tune the yield function. Although, in general, BCC materials could have $\{110\},\{112\}$ and $\{123\}$ slip 330 planes, in the non-Schmid crystal plasticity model only $\{1 \overline{1} 0\}$ planes of $\langle 111\rangle$ zone are considered. Table 1 lists the slip systems available in the non-Schmid model [17]. 
Table 1: This table list the slip systems available in the non-Schmid crystal Plasticity model [17.

\begin{tabular}{|c|c|c|c|c|c|c|c|c|c|}
\hline$\alpha$ & Reference system & $m^{\alpha}$ & $n^{\alpha}$ & $n_{1}^{\alpha}$ & $\alpha$ & Reference system & $m^{\alpha}$ & $n^{\alpha}$ & $n_{1}^{\alpha}$ \\
\hline 1 & $(01 \overline{1})[111]$ & {$[111]$} & {$[01 \overline{1}]$} & {$[\overline{1} 10]$} & 13 & $(01 \overline{1})[\overline{1} \overline{1} \overline{1}]$ & {$[\overline{1} \overline{1} \overline{1}]$} & {$[01 \overline{1}]$} & {$[10 \overline{1}]$} \\
\hline 2 & $(\overline{1} 01)[111]$ & {$[111]$} & {$[\overline{1} 01]$} & {$[0 \overline{1} 1]$} & 14 & $(\overline{1} 01)[\overline{1} \overline{1} \overline{1}]$ & {$[\overline{1} \overline{1} \overline{1}]$} & {$[\overline{1} 01]$} & {$[\overline{1} 10]$} \\
\hline 3 & $(1 \overline{1} 0)[111]$ & {$[111]$} & {$[1 \overline{1} 0]$} & {$[10 \overline{1}]$} & 15 & $(1 \overline{1} 0)[\overline{1} \overline{1} \overline{1}]$ & {$[\overline{1} \overline{1} \overline{1}]$} & {$[1 \overline{1} 0]$} & {$[0 \overline{1} 1]$} \\
\hline 4 & $(\overline{1} 0 \overline{1})[\overline{1} 11]$ & {$[\overline{1} 11]$} & {$[\overline{1} 0 \overline{1}]$} & {$[\overline{1} \overline{1} 0]$} & 16 & $(\overline{1} 0 \overline{1})[\overline{1} \overline{1} \overline{1}]$ & {$[1 \overline{1} \overline{1}]$} & {$[\overline{1} 0 \overline{1}]$} & {$[01 \overline{1}]$} \\
\hline 5 & $(0 \overline{1} 1)[\overline{1} 11]$ & {$[\overline{1} 11]$} & {$[0 \overline{1} 1]$} & {$[101]$} & 17 & $(0 \overline{1} 1)[1 \overline{1} \overline{1}]$ & {$[1 \overline{1} \overline{1}]$} & {$[0 \overline{1} 1]$} & {$[\overline{1} \overline{1} 0]$} \\
\hline 6 & $(110)[\overline{1} 11]$ & {$[\overline{1} 11]$} & {$[110]$} & {$[01 \overline{1}]$} & 18 & $(110)[1 \overline{1} \overline{1}]$ & {$[1 \overline{1} \overline{1}]$} & {$[110]$} & {$[101]$} \\
\hline 7 & $(0 \overline{1} \overline{1})[\overline{1} \overline{1} 1]$ & {$[\overline{1} \overline{1} 1]$} & {$[0 \overline{1} \overline{1}]$} & {$[1 \overline{1} 0]$} & 19 & $(0 \overline{1} \overline{1})[11 \overline{1}]$ & {$[11 \overline{1}]$} & {$[0 \overline{1} \overline{1}]$} & {$[\overline{1} 0 \overline{1}]$} \\
\hline 8 & $(101)[\overline{1} \overline{1} 1]$ & {$[\overline{1} \overline{1} 1]$} & {$[101]$} & {$[011]$} & 20 & $(101)[11 \overline{1}]$ & {$[11 \overline{1}]$} & {$[101]$} & {$[1 \overline{1} 0]$} \\
\hline 9 & $(\overline{1} 10)[\overline{1} \overline{1} 1]$ & {$[\overline{1} \overline{1} 1]$} & {$[\overline{1} 10]$} & {$[\overline{1} 0 \overline{1}]$} & 21 & $(\overline{1} 10)[11 \overline{1}]$ & {$[11 \overline{1}]$} & {$[\overline{1} 10]$} & {$[011]$} \\
\hline 10 & $(10 \overline{1})[1 \overline{1} 1]$ & {$[1 \overline{1} 1]$} & {$[10 \overline{1}]$} & {$[110]$} & 22 & $(10 \overline{1})[\overline{1} 1 \overline{1}]$ & {$[\overline{1} 1 \overline{1}]$} & {$[10 \overline{1}]$} & {$[0 \overline{1} \overline{1}]$} \\
\hline 11 & $(011)[1 \overline{1} 1]$ & {$[1 \overline{1} 1]$} & {$[011]$} & {$[\overline{1} 01]$} & 23 & $(011)[\overline{1} 1 \overline{1}]$ & {$[\overline{1} 1 \overline{1}]$} & {$[011]$} & {$[110]$} \\
\hline 12 & $(\overline{1} \overline{1} 0)[1 \overline{1} 1]$ & {$[\overline{1} \overline{1} 1]$} & {$[\overline{1} \overline{1} 0]$} & {$[0 \overline{1} \overline{1}]$} & 24 & $(\overline{1} \overline{1} 0)[\overline{1} 1 \overline{1}]$ & {$[\overline{1} 1 \overline{1}]$} & {$[\overline{1} \overline{1} 0]$} & {$[\overline{1} 01]$} \\
\hline
\end{tabular}




\section{Modeling the hardening rate of a single crystal}

Shear stress shear strain curves for single crystals that are initially oriented for single slip show three distinct stages usually referred to as Stage I, II and Stage III hardening 29. The hardening rate just after yielding is low. Most of the deformation in this stage is accommodated by the primary slip system and hardening is chiefly due to self-hardening. The low hardening rate corresponds to a large dislocation mean free path and few barriers. This stage I regime is also known as the "easy glide" [29].

The second stage, which is known as "linear hardening", starts when other slip systems become activated due to crystal rotation during deformation and interact with the primary slip system. This reduces the mean free path of dislocations and considerably increases the hardening rate. During this stage the dislocation density increases [29]. The hardening rate eventually decreases which signals the onset of the third stage, "exhaustion hardening". The high stress and dislocation density activates cross slip and dynamic recovery which in turn softens the material.

Although the classical Hill type hardening rule, equation (29), is widely used in crystal plasticity codes to model the hardening behavior of polycrystalline metals, it can only model the stress-strain behavior observed in the first stage of the deformation of a single crystal. The hardening rate predicted by equation 29) does not change significantly during the deformation.

As was discussed above, the hardening rate in each stage of the deformation of a single crystal is controlled by a different set of underlying mechanisms. Equation (31) effectively only models those mechanisms that are active in the first stage, and predicts a rather uniform increase in hardening. To capture the variable hardening rate of a single crystal, a more sophisticated model is needed.

\subsection{The Differential-Exponential (DE) hardening model}

A single crystal oriented for single slip typically hardens linearly with a small slope in the first stage. In the second stage, the density of mobile dislocations 
decreases exponentially [37, which results in an exponential increase in the hardening of material. To model this behavior, the authors propose the following exponential equation for self-hardening:

$$
h_{\text {stage } 2}^{\beta}=h_{0 D E} \exp \left[\gamma^{1 s t}+\left(1+\gamma^{1 s t}\right)^{\nu}\right]
$$

365 strain on the most active (primary) slip system, and exponent $\nu$ is a material parameter. As will be discussed later in this section, the above equation successfully predicts the two-stage stress-strain behavior of a single crystal. However, its definition is incomplete without some criteria for predicting the initiation of

the following self-hardening equation for stages I and II in a single crystal is

Based on the knowledge that second stage hardening occurs in single crystals when secondary slip systems become active, it became evident that criteria for the start of the second stage hardening must be a function of the ratio of the accumulated shear strain and critical resolved shear stresses in primary and 375 secondary slip systems. The reason for the activation of secondary slip systems is the eventual rotation of the primary slip system away from the loading direction, caused by the evolution of texture induced by increased plastic deformation.

Using the definition of a step function:

$$
\langle x\rangle= \begin{cases}0, & x<0 \\ 1, & x \geq 0\end{cases}
$$

proposed. Due to its ability to differentiate between active slip systems, as well as the use of the exponential function, this new hardening model was termed the Differential-Exponential (DE) hardening model.

$$
h_{D E}^{\beta}=\left(h_{\text {stage } 1}^{\beta}-h_{\text {stage } 2}^{\beta}\right)\left\langle k_{1}-\frac{\max \left(\tau^{\beta}\right)}{\tau_{s}}\right\rangle\left\langle k_{2}-\frac{\gamma^{2 n d}}{\gamma^{1 s t}}\right\rangle+h_{\text {stage } 2}^{\beta}
$$

In this equation, $k_{1}$ and $k_{2}$ are material parameters to be fitted to the experimental stress-strain curve, $\tau^{\beta}$ is the slip resistance on a slip system $\beta$, and 
$385 \max \left(\tau^{\beta}\right)$ is the largest slip resistance among all slip systems, $\tau_{s}$ is the saturation value, $\gamma^{1 s t}$ and $\gamma^{2 n d}$ are the cumulative shear strains on the first and second most active slip systems. It should also be noted that at the start of deformation when the value of both step functions is equal to 1 , equation 35 reduces to $h_{\text {stage } 1}^{\beta}$, which is the Hill's hardening equation 29 . However, as

390 the plastic deformation increases and the value of either of those two step functions becomes zero, the second stage hardening, $h_{\text {stage } 2}^{\beta}$, becomes activated. It should be pointed out that when the values of $k_{1}$ and $k_{2}$ are very large, the step functions become equal to 1 , and equation 35 behaves similar to equation (31).

The self-hardening rate equation (31) was replaced by equation 35 in the non-Schmid crystal plasticity model, and implemented in the commercial FEM software Abaqus ${ }^{\circledR}$ as a user defined material subroutine (VUMAT).

\subsection{Stress-integration algorithm to solve for shear rate}

This section summarizes the algorithm for stress integration which follows [30]. This algorithm is developed for the Differential-Exponential hardening rule.

1. The following variables are assumed to be known at the beginning of an increment i for a grain $\left(\hat{\sigma}_{i}, g_{i}^{\alpha}, \Delta \hat{\varepsilon}_{i}, Q_{i}\right)$ in the element coordinate system. Parameter $Q_{i}$ is the crystal orientation matrix. Then stress and strain tensors are rotated to the crystal coordinate system as:

$$
\begin{gathered}
\sigma_{i}=Q_{i} \hat{\sigma}_{i} Q_{i}^{T} \\
\Delta \varepsilon_{i}=Q_{i} \Delta \hat{\varepsilon}_{i} Q_{i}^{T}
\end{gathered}
$$

2. Initialize variables

$i$ : FEM increment

$k$ : Plasticity iteration 


$$
\begin{aligned}
\sigma_{i+1}^{(0)} & =\sigma_{i}+C^{e}: \Delta \varepsilon_{i} \\
g_{i+1}^{\alpha(0)} & =g_{i}^{\alpha} \\
\Delta \lambda_{i+1}^{(0)} & =0 \\
k & =1
\end{aligned}
$$

3. Evaluate the yield condition $Y_{i+1}^{(k)}$

$$
\begin{aligned}
Y_{i+1}^{(k)} & =Y\left(\sigma^{(k-1)}, u^{(k-1)}\right)=\frac{1}{\rho_{2}} \ln \left\{\sum_{\alpha=1}^{N} \exp \left[\frac{\rho_{2}}{m_{2}}\left(\frac{\left|\sigma: d^{* \alpha}\right|}{g_{y}^{\alpha}}-1\right)\right]\right\} \\
d^{* \alpha} & =d^{\alpha}+\sum_{\eta} a_{\eta}^{\alpha} d_{\eta}^{\alpha} \\
d^{\alpha} & =\frac{1}{2}\left(m^{\alpha} \otimes n^{\alpha}+n^{\alpha} \otimes m^{\alpha}\right) \\
\sum_{\eta} a_{\eta}^{\alpha} d_{\eta}^{\alpha} & =a_{1} m^{\alpha} \otimes n_{1}^{\alpha}+a_{2}\left(n^{\alpha} \times m^{\alpha}\right) \otimes n^{\alpha}+a_{3}\left(n_{1}^{\alpha} \times m^{\alpha}\right) \otimes n_{1}^{\alpha} \\
& +a_{4} n^{\alpha} \otimes n^{\alpha}+a_{5}\left(n^{\alpha} \times m^{\alpha}\right) \otimes\left(n^{\alpha} \times m^{\alpha}\right)+a_{6} m^{\alpha} \otimes m^{\alpha}
\end{aligned}
$$

If $Y_{i+1}^{(k)} \leq 0$ go to step 10

4. Compute the flow potential

$$
\begin{aligned}
F_{i+1}^{(k)} & =F\left(\sigma^{(k-1)}, u^{(k-1)}\right)=\frac{1}{\rho_{1}} \ln \left\{\sum_{\alpha=1}^{N} \exp \left[\frac{\rho_{1}}{m_{1}}\left(\frac{\left|\sigma: d^{\alpha}\right|}{g_{y}^{\alpha}}-1\right)\right]\right\} \\
d^{\alpha} & =\frac{1}{2}\left(m^{\alpha} \otimes n^{\alpha}+n^{\alpha} \otimes m^{\alpha}\right)
\end{aligned}
$$

5. Calculate the correction to the plastic consistency parameter

$$
\delta \Delta \lambda_{i+1}^{(k)}=\frac{Y_{i+1}^{(k)}}{\frac{\partial Y_{i+1}^{(k)}}{\partial \sigma}: C^{e}: \frac{\partial F_{i+1}^{(k)}}{\partial \sigma}-\sum_{\alpha=1}^{N} \sum_{\beta=1}^{N} h^{\alpha \beta} \operatorname{sgn}(\Delta \lambda) \frac{\partial Y_{i+1}^{(k)}}{\partial \tau^{\alpha}}\left|\frac{\partial F_{i+1}^{(k)}}{\partial \tau^{\beta}}\right|}
$$

6. Compute stress, slip resistance, and yield function 


$$
\begin{aligned}
\Delta \sigma_{i+1}^{(k)} & =\Delta \sigma_{i+1}^{(k-1)}-\delta \Delta \lambda_{i+1}^{(k)} C^{e}: \frac{\partial F\left(\sigma_{i+1}^{(k-1)}, g_{i+1}^{(k-1)}\right)}{\partial \sigma} \\
\delta \Delta \gamma_{i+1}^{\alpha(k)} & =\delta \Delta \lambda_{i+1}^{(k)} \frac{\partial F\left(\sigma_{i+1}^{(k-1)}, g_{i+1}^{(k-1)}\right)}{\partial \tau^{\alpha}}
\end{aligned}
$$

If $\left.k_{1}>\frac{\max \left(\tau^{\beta}\right)}{\tau_{s}}\right\rangle$ and $\left\langle k_{2}>\frac{\gamma^{2 n d}}{\gamma^{1 s t}}\right\rangle$

$$
\begin{array}{r}
\delta \Delta g_{i+1}^{\alpha(k)}=\sum_{\beta=1}^{N} h_{0}\left|1-\frac{g_{i+1}^{\beta(k-1)}}{\tau_{s}}\right|^{\eta} \operatorname{sgn}\left(1-\frac{g_{i+1}^{\beta(k-1)}}{\tau_{s}}\right) \\
{\left[q+(1-q) \delta^{\alpha \beta}\right]\left|\delta \Delta \lambda_{i+1}^{(k)} \frac{\partial F\left(\sigma_{i+1}^{(k-1)}, g_{i+1}^{(k-1)}\right)}{\partial \tau^{\alpha}}\right|}
\end{array}
$$

415

Else,

$$
\begin{aligned}
\delta \Delta g_{i+1}^{\alpha(k)} & =\sum_{\beta=1}^{N} h_{0 D E} \exp \left[\gamma^{1 s t}+\left(1+\gamma^{1 s t}\right)^{\nu}\right] \\
& {\left[q+(1-q) \delta^{\alpha \beta}\right]\left|\delta \Delta \lambda_{i+1}^{(k)} \frac{\partial F\left(\sigma_{i+1}^{(k-1)}, g_{i+1}^{(k-1)}\right)}{\partial \tau^{\alpha}}\right| }
\end{aligned}
$$

Then

$$
g_{i+1}^{\alpha(k)}=g_{i+1}^{\alpha(k-1)}+\delta \Delta g_{i+1}^{\alpha(k)}
$$

7. If $Y_{i+1}^{(k)} \leq$ Tolerance go to step 9 , otherwise go to step 8 .

8. Update the consistency parameter

$$
\begin{aligned}
\Delta \lambda_{i+1}^{\alpha(k)} & =\Delta \lambda_{i+1}^{\alpha(k-1)}+\delta \Delta \lambda_{i+1}^{\alpha(k)} \\
k & =k+1
\end{aligned}
$$

Then Go to 3 , 
440

\section{CPFE modeling of uniaxial compression of QP980 ferrite micropil- lar}

The models developed in sections 3 and 4 were used to predict the deforma-

First, the experimental results from compression of QP980 ferrite micropillars are presented, and then they are compared with the predictions of Schmid and non-Schmid crystal plasticity models.

\subsection{Uniaxial compression of QP980 ferrite micropillars}

Six ferrite micropillars were carved out of the bulk of QP980 steel using Focused Ion Beam (FIB), which were then named 1-6, respectively. Each micropillar was extracted from a ferrite grain with a distinct initial orientation. Figure 9 shows the orientation of the compression axis of these micropillars in an inverse pole figure. Figure 2 shows a schematic of the cylindrical micropillar with a taper angle of $4^{\circ}$, a height to diameter ratio of $2-3$, and top surface diameter between $1.0 \mu \mathrm{m}$ and $1.7 \mu \mathrm{m}$. The micropillar compression test was performed with a flat punch nano-indenter, and the force-displacement data was recorded for each experiment. More details on these experiments are given in [27.

Slip bands were observed at the end of the deformation of some of these micropillars. These bands changed the effective cross section of micropillars, which rendered calculation of engineering stress inappropriate. Therefore, the experimental data in this paper will be presented as force-displacement curves.

\subsection{Modeling QP980 ferrite micropillars}

The geometry of micropillars were modeled in Abaqus ${ }^{\circledR}$ commercial finite element software. The flat punch nano-indenter was modeled with a rigid surface. 
A friction coefficient of 0.5 was applied between the punch and the micropillar to prevent the slip between the surfaces [25]. The base of each micropillar was modeled with a perfectly elastic material to approximate the elastic deformation

Both Schmid and non-Schmid crystal plasticity models were implemented into Abaqus as user defined material subroutines (UMAT) and were used to predict the deformation behavior of these micropillars. In the crystal plasticity finite element simulations, the typical 48 slip systems for BCC materials, i.e.

${ }_{455}\{110\},\{112\},\{123\}$ planes and $\langle 11 \overline{1}\rangle$ slip directions, were used in the Schmid case. These will be referred to as slip family 1 to 3 respectively. In the nonSchmid case, the 24 slip systems (12 slip systems of $\{110\}\langle 11 \overline{1}\rangle$ in positive and 12 in negative slip direction) shown in Table 1 were used. Since in this case the normal stresses also affect the yield stress, the positive and negative sense of the slip translates to tensile and compressive stresses, which in turn affect the onset of dislocation slip through the non-Schmid effect.

\subsection{Material parameter fitting and evaluation}

The criterion for evaluating the quality of the parameter fit was based on how accurately the calibrated CPFE model predicted the uniaxial compressive forcedisplacement curve of the remaining micropillars with distinct orientations. The metric used to quantify the accuracy of the fit was based on the calculated error between experimental and predicted force-displacement curves, using equation A.2 in the Appendix A The consistency of the fit was evaluated based on the variation in the calculated error for all available experimental data.

In the Schmid model, $\tau_{S i}, \tau_{0 i}$ and $h_{0 i}$ (where $i=1 \ldots 3$ refers to the slip family) were used as fitting parameters. These are the saturation shear stress, initial critical resolved shear stress and hardening rate, respectively. In the non-Schmid model, $\tau_{S}, \tau_{0}, h_{0}$ and $a_{i}$ (where $i=1 \ldots 3$ ) were used as fitting parameters. Here, $a_{i}$ are the non-Schmid constants. The hardening exponent ${ }_{475} \eta_{i}$ (where $i=1 \ldots 3$ ) in the Schmid model and $\eta$ in the non-Schmid model were kept constant, $\eta_{i}=\eta=3$. Also, the value of the initial hardening in the 
non-Schmid model was assumed to be a constant, $h_{0}=10$.

Material parameters for Schmid and non-Schmid models were fitted using one or more experimental force-displacement curves through an iterative procedure using commercial optimization software LS-OPT ${ }^{\circledR}$. More details about the model calibration process is provided in the Appendix A and in [38.

In the case of the non-Schmid model, it was found that a minimum of two experimental force-displacement curves (with different crystal orientations) are needed to get an accurate and consistent fit. When only one force-displacement curve was used, the fitted parameters were inconsistent and the non-Schmid CPFE model was unable to accurately predict the force-displacement curve for other micropillar orientations. Further improvement with using more than two experimental force-displacement curves was negligible. Also, it was found that the non-Schmid parameters, $a_{4}, a_{5}$ and $a_{6}$ have negligible effect on the predictions of the model, therefore, they were ignored and only $a_{1}, a_{2}$ and $a_{3}$ were considered in the non-Schmid model.

In the case of the Schmid model, it was discovered that the accuracy of this model's prediction is less than that of the non-Schmid model, regardless of how many experimental force-displacement curves were used for parameter calibration. The accuracy and consistency of the Schmid model, however, improved when two or more experimental force-displacement curves were used in the calibration.

Material parameters fitted using various combinations of force-displacement curves are shown in Tables A.1 and A.3 for the non-Schmid model, and Tables ${ }_{500}$ A.5 and A.7 for the Schmid model, in the Appendix A.

\subsection{Predictions of calibrated models}

In this section, only select number of CPFE simulation results will be presented and discussed. The summary of all numerical simulation results and their comparison with experimental data are given in the Appendix A. Table 2 shows the fitted material parameters for the Schmid model using two micropillars (i.e. 1 and 4 ), and Table 3 shows the fitted material parameters for the 
non-Schmid model using three micropillar force-displacement curves (i.e., 1, 3 and 5). The CPFE simulation results based on these fitted parameters will be discussed next.

Figures 3 through 8 show the experimental force-displacement curves for micropillars 1-6. For comparison purposes, the force-displacement curves predicted by the Schmid model calibrated with micropillars 1 and 4 , and those predicted by the non-Schmid model calibrated with micropillars 1, 3 and 5 are also shown in the same figures.

Overall, it can be seen from Figures 4, 5, 7, and 8 , that the force-displacement predictions of the Schmid model for micropillars 2, 3, 5 and 6 are erratic. That is, in some cases the Schmid model overestimates, and in other cases it underestimates the experimental curve. This erratic prediction by the Schmid model, however, is not unexpected as ferrite has a BCC crystal structure, and its deformation is best described by the non-Schmid law. This will be discussed in more detail in the next section. Tables A.6 and A.8 in the Appendix A show the magnitude of error and sign (i.e. over or underestimation) associated with different different calibrations of the Schmid model. For example, Table A.8 shows that the Schmid model calibrated with micropillars 1 and 4 underestimate the experimental curve for micropillar 5 by $11.93 \%$ (see Figure 7), and overestimate that for micropillar 3 by $27.93 \%$ (see Figure 5 ). The worst overall prediction by the Schmid model is for micropillar 3 with an error of $47.75 \%$, when the model was calibrated with the single force-displacement curve from micropillar 5 (Table A.6).

The non-Schmid model's predictions, however, are much more consistent and closer to experimental force-displacement curves. Table A.4 in the Appendix A shows that the worst prediction of the non-Schmid model calibrated with micropillars 1,3 and 5 is for micropillar 5 with an error value of -8.98 , which implies that it underestimated the experimental curve by $8.98 \%$ (see Figure 7 ).

535 Finally, Table A.2 shows that the non-Schmid model's worst overall error is for micropillar 3 with an error of $44.73 \%$, when the model is calibrated with a single force-displacement curve from micropillar 6 . 


\subsection{Further discussions on Schmid versus non-Schmid law}

As explained in Section 3, the non-Schmid model considers the effect of stresses that are applied on planes other than the slip plane or along directions other than the slip direction. Depending on the orientation of the material, these stresses can contract the dislocation core and reduce the critical stress needed for yielding. Similarly, for some other orientations the combined effect of the non-Schmid stresses can expand the dislocation core and increase the stress needed for the initiation of dislocation slip and yielding. This is the reason why, compared with the Schmid model, the non-Schmid model can more consistently and accurately predict the force-displacement curves of all micropillars.

The deviation from Schmid law can be further analyzed using the following equation which represents the ratio of the non-Schmid resolved shear stress, equation (17), to the Schmid resolved shear stress [26].

$R_{n s}=\frac{\sigma:\left(m^{\alpha} \otimes n^{\alpha}\right)+a_{1} \sigma:\left(m^{\alpha} \otimes n_{1}^{\alpha}\right)+a_{2} \sigma:\left[\left(n^{\alpha} \times m^{\alpha}\right) \otimes n^{\alpha}\right]+a_{3} \sigma:\left[\left(n_{1}^{\alpha} \times m^{\alpha}\right) \otimes n_{1}^{\alpha}\right]}{\sigma:\left(m^{\alpha} \otimes n^{\alpha}\right)}$

When this ratio is equal to 1.0, the material behaves according to Schmid law. In contrast, the more it deviates from 1.0, the more the material behavior deviates from the Schmid type. Figure 9 shows the orientation of the compression axis for QP980 ferrite micropillars 1-6 mapped over the contour of $R_{n s}$ as defined by equation (36). The calculated value of $R_{n s}$ for each micropillar is also shown in Table 4

When the Schmid model is calibrated with the force-displacement data of a material with a given $R_{n s}^{*}$, the model will overestimate the force-displacement of all other orientations with $R_{n s}>R_{n s}^{*}$, and underestimate those with $R_{n s}<$ $R_{n s}^{*}$. For example, in Figures 3 through 8 , the Schmid model was calibrated with micropillars 1 and 4 , which have $R_{n s}$ of 1.09 and 1.29 , respectively. In comparison, micropillars 2 and 3 have higher $R_{n s}$ of 1.33 and 1.78 (see Table 4), which means that the contribution of non-Schmid stresses to yielding for these micropillars is greater than those for micropillars 1 and 4 . In contrast, 
Table 2: Material parameters found for the Schmid model (using QP980 Ferrite micropillars 1 and 4$)$.

\begin{tabular}{|c|c|c|c|c|c|c|c|c|c|c|c|}
\hline$\tau_{S 1}$ & $\tau_{S 2}$ & $\tau_{S 3}$ & $\tau_{01}$ & $\tau_{02}$ & $\tau_{03}$ & $h_{01}$ & $h_{02}$ & $h_{03}$ & $\eta_{1}$ & $\eta_{2}$ & $\eta_{3}$ \\
\hline 650 & 650 & 720 & 195 & 205 & 330 & 110 & 110 & 110 & 3 & 3 & 3 \\
\hline
\end{tabular}

Table 3: Material parameters found for the non-Schmid model (using QP980 Ferrite micropillars 1,3 and 5).

\begin{tabular}{|c|c|c|c|c|c|c|}
\hline$\tau_{S}$ & $\tau_{0}$ & $h_{0}$ & $\eta$ & $a_{1}$ & $a_{2}$ & $a_{3}$ \\
\hline 537 & 249 & 10 & 3 & 0.0103 & 0.5748 & 0.0007 \\
\hline
\end{tabular}

micropillars 5 and 6 have lower $R_{n s}$ of 0.96 and 0.94 , which implies that the contribution of the non-Schmid stresses to their yielding is less than those for micropillars 1 and 4. Therefore, in the absence of non-Schmid stresses, the Schmid model calibrated with micropillars 1 and 4 will overestimate the required critical resolved shear stress (CRSS) to deform micropillars 2 and 3, and in turn overestimates their experimental force-displacement curve (as seen in Figures 4 and 5). On the other hand, given that micropillars 5 and 6 have smaller $R_{n s}$ values, the same Schmid model underestimates their CRSS, and in turn underestimates their experimental force-displacement curves (as seen in Figures 7 and 87 .

Figure 10 further confirms this conclusion. This figure compares the predictions of the Schmid model for micropillar 2 when the model is calibrated with pairs of micropillars 1 and 6 and micropillars 4 and 5 . The $R_{n s}$ value for mircopillar 2 is greater than that for micropillars 1, 4, 5 and 6 which were used to calibrate the Schmid model. As expected, both calibrations overestimate the behavior of micropillar 2 .

Table 4: Value of deviation from Schmid type behavior $R_{n s}$ as defined in equation 36 .

\begin{tabular}{|c|c|c|c|c|c|c|}
\hline Micropillar & 1 & 2 & 3 & 4 & 5 & 6 \\
\hline$R_{n s}$ & 1.09 & 1.33 & 1.78 & 1.29 & 0.96 & 0.94 \\
\hline
\end{tabular}




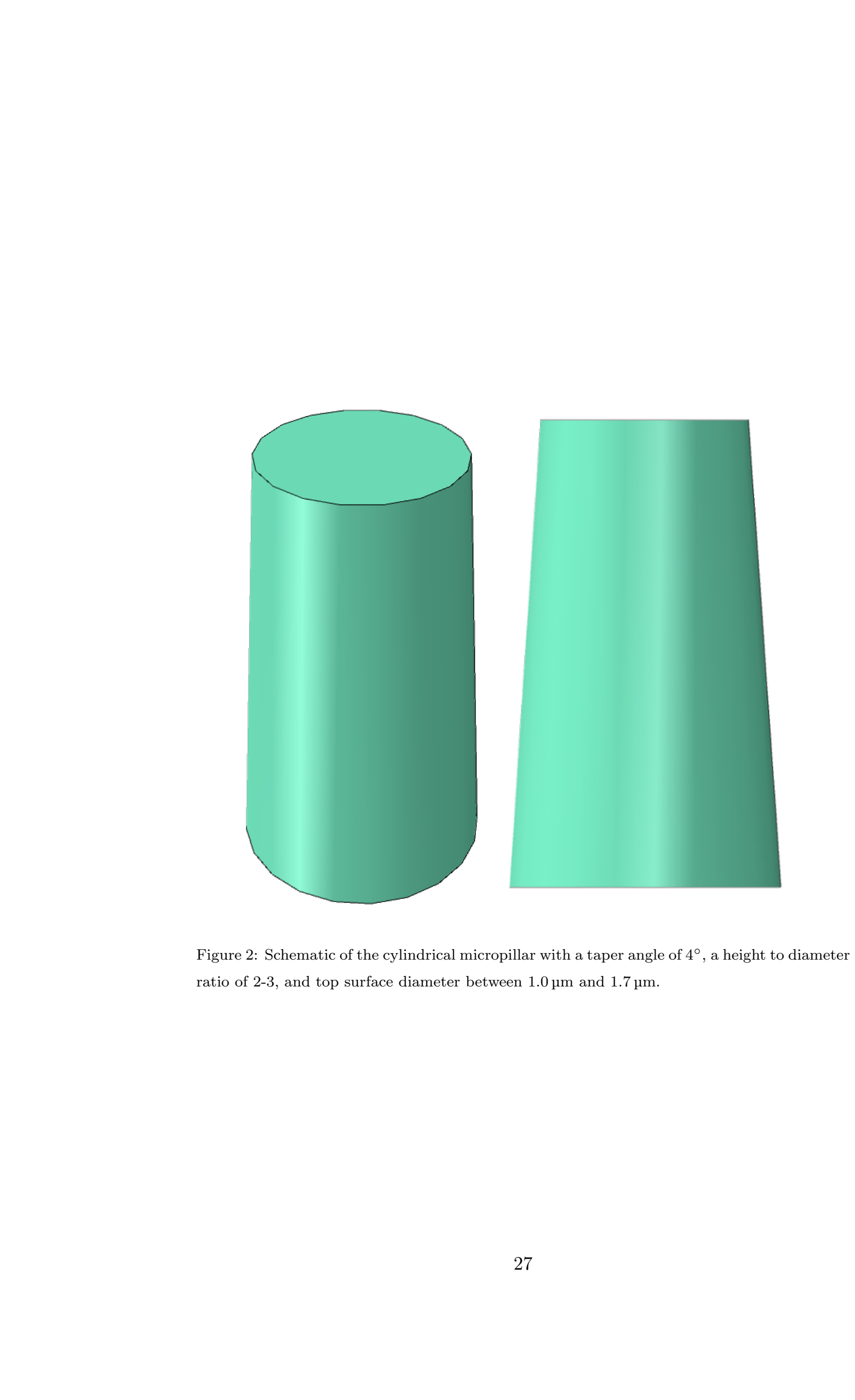




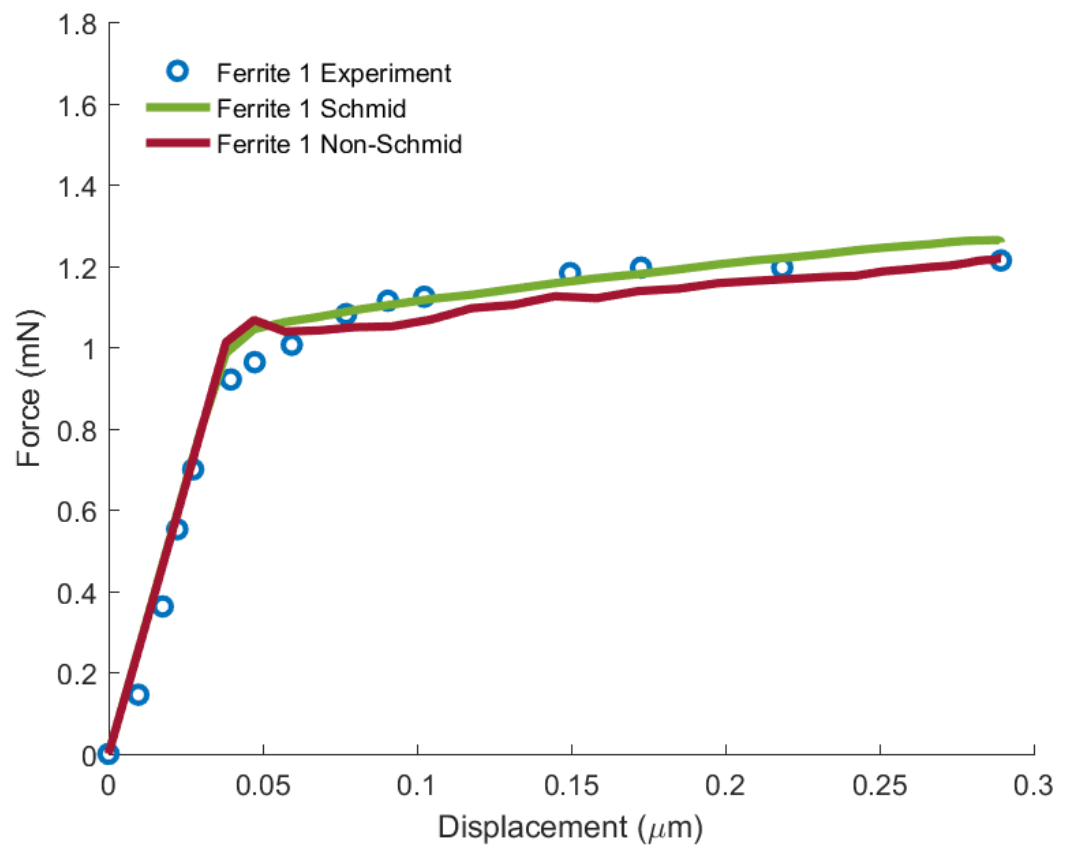

Figure 3: The green curve shows the Schmid and the red curve shows the non-Schmid models fit to ferrite micropillar 1 . The Schmid model was simultaneously fitted to micropillars 1 and 4, while the non-Schmid model was calibrated with micropillars 1, 3 and 5 . The blue circles represent the experimental measurements. 


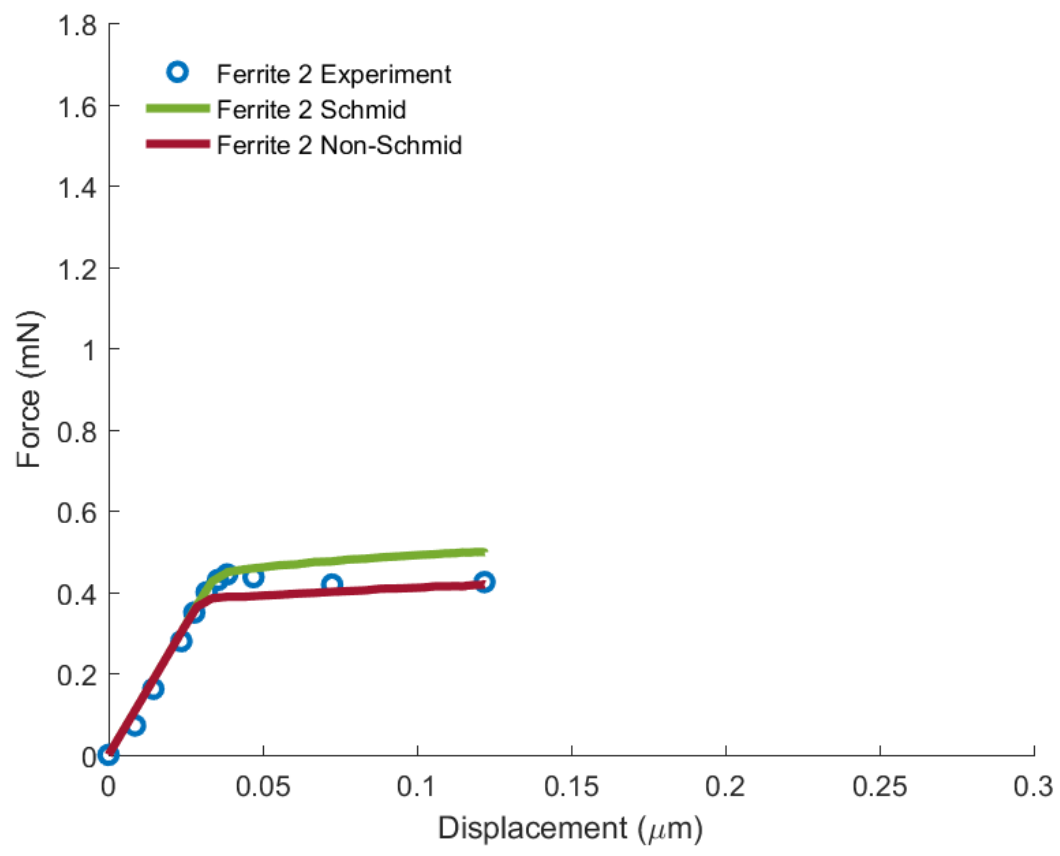

Figure 4: Comparing predictions of Schmid (green curve) and non-Schmid (red curve) models for ferrite micropillar 2 against the experiment. The Schmid model was simultaneously fitted to micropillars 1 and 4, while the non-Schmid model was calibrated with micropillars 1, 3 and 5. 


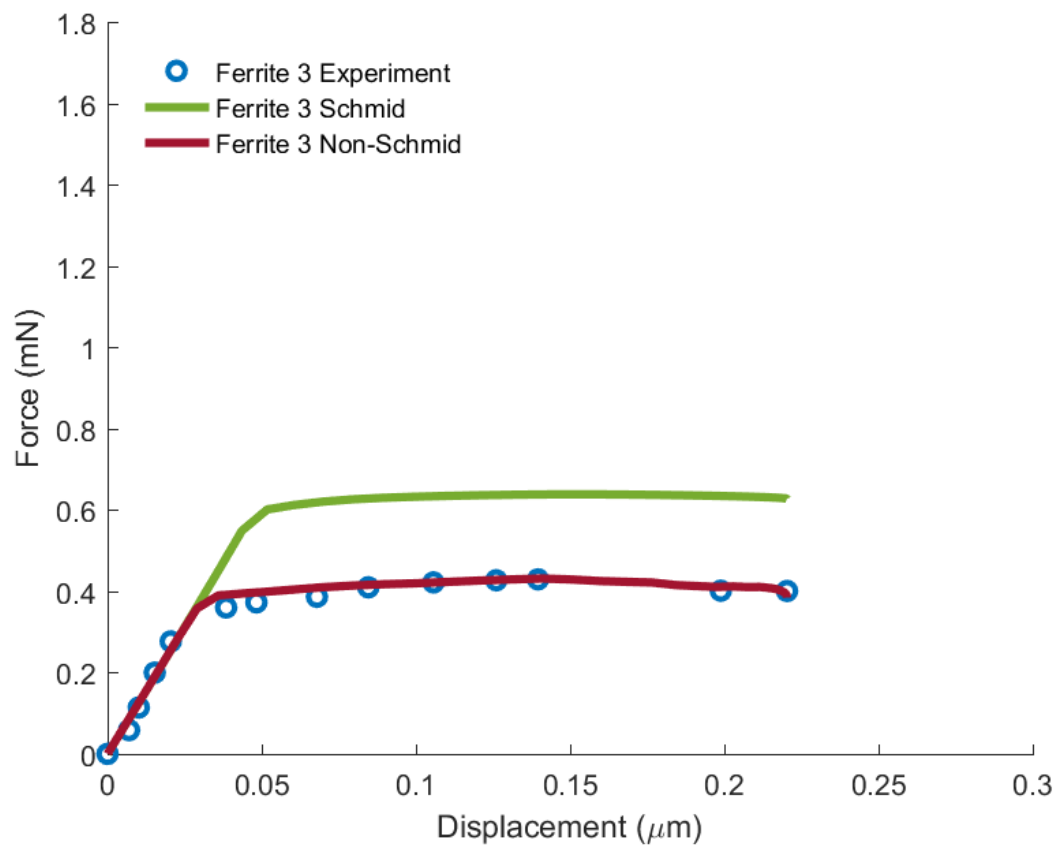

Figure 5: Calibration of the non-Schmid model (red curve) to micropillar 3. The non-Schmid model was simultaneously calibrated with micropillars 1, 3 and 5 . This figure also compares the predictions of the Schmid model (green curve) for ferrite micropillar 3 against the experiment. The Schmid model was simultaneously fitted to micropillars 1 and 4 . 


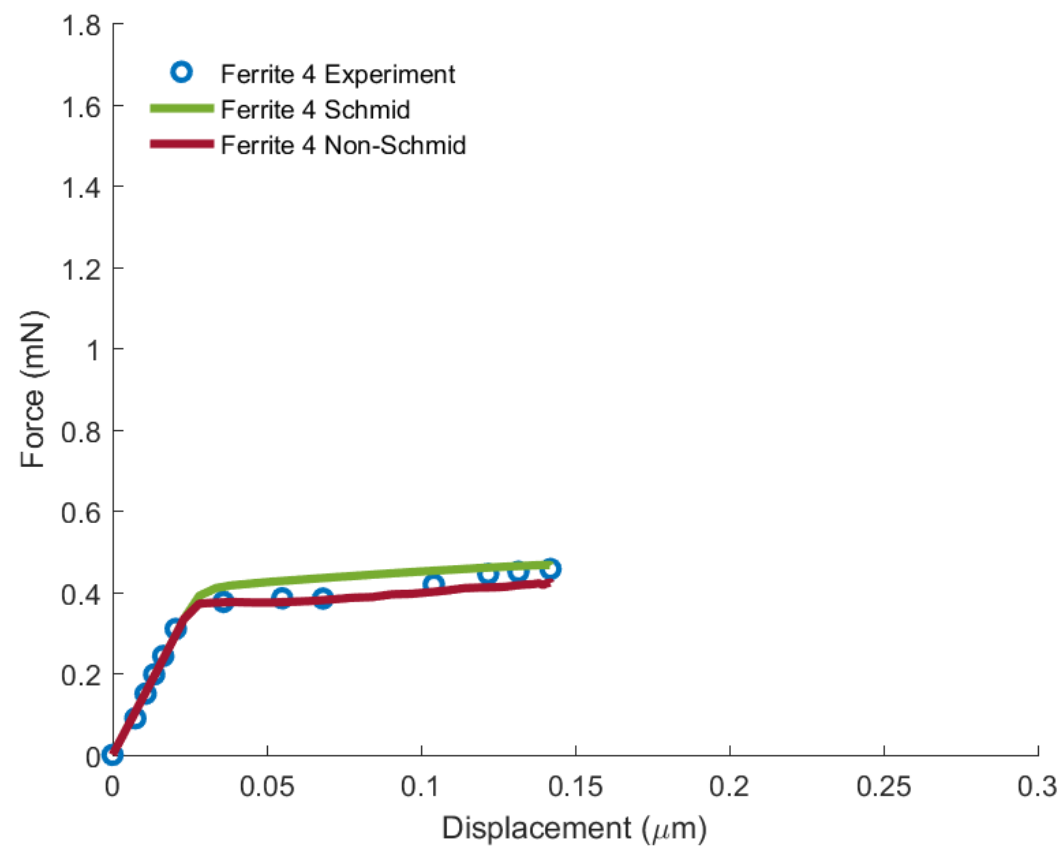

Figure 6: The green curve shows the calibration of the Schmid model to micropillar 4. The red curve shows the prediction of the non-Schmid model for ferrite micropillar 4 against the experiment. The Schmid model was simultaneously fitted to micropillars 1 and 4 , while the non-Schmid model was calibrated with micropillars 1, 3 and 5. 


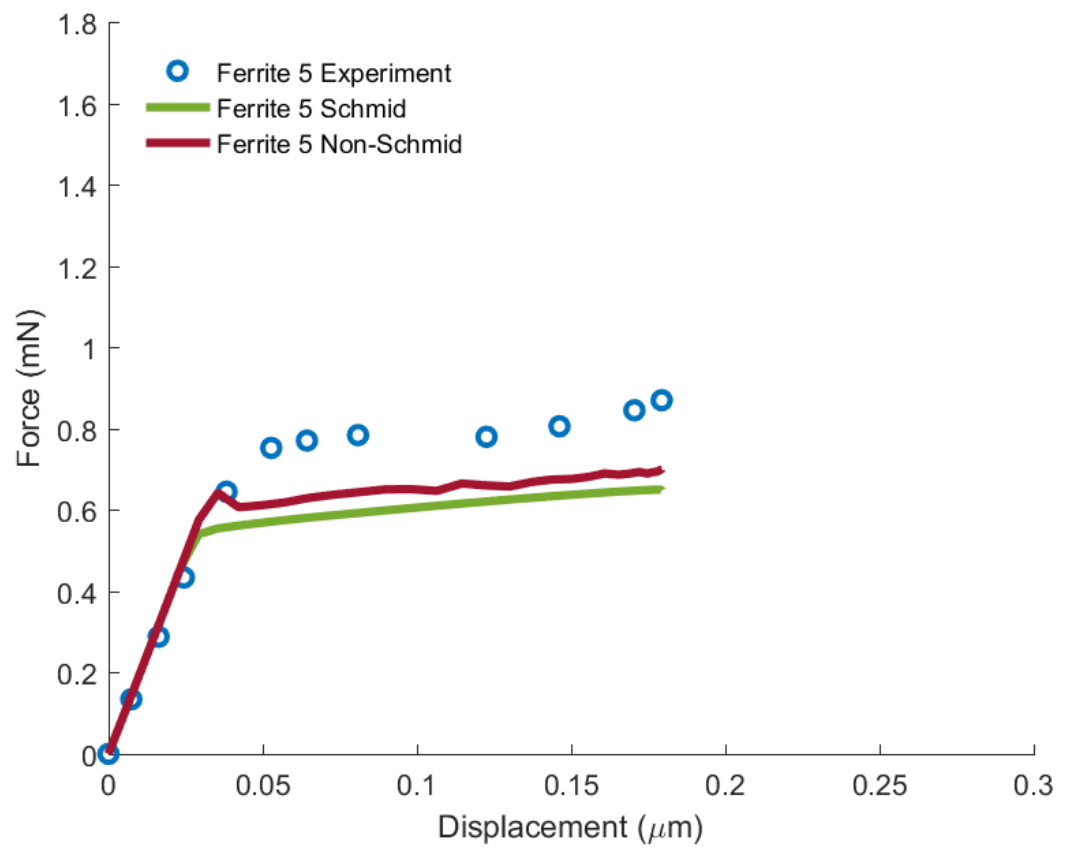

Figure 7: The red curve shows the fitting of the non-Schmid model to micropillar 5. This model was simultaneously calibrated with micropillars 1,3 and 5 . The green curve shows the prediction of the Schmid model for ferrite micropillar 5, based on material parameters fit to curves 1 and 4 . 


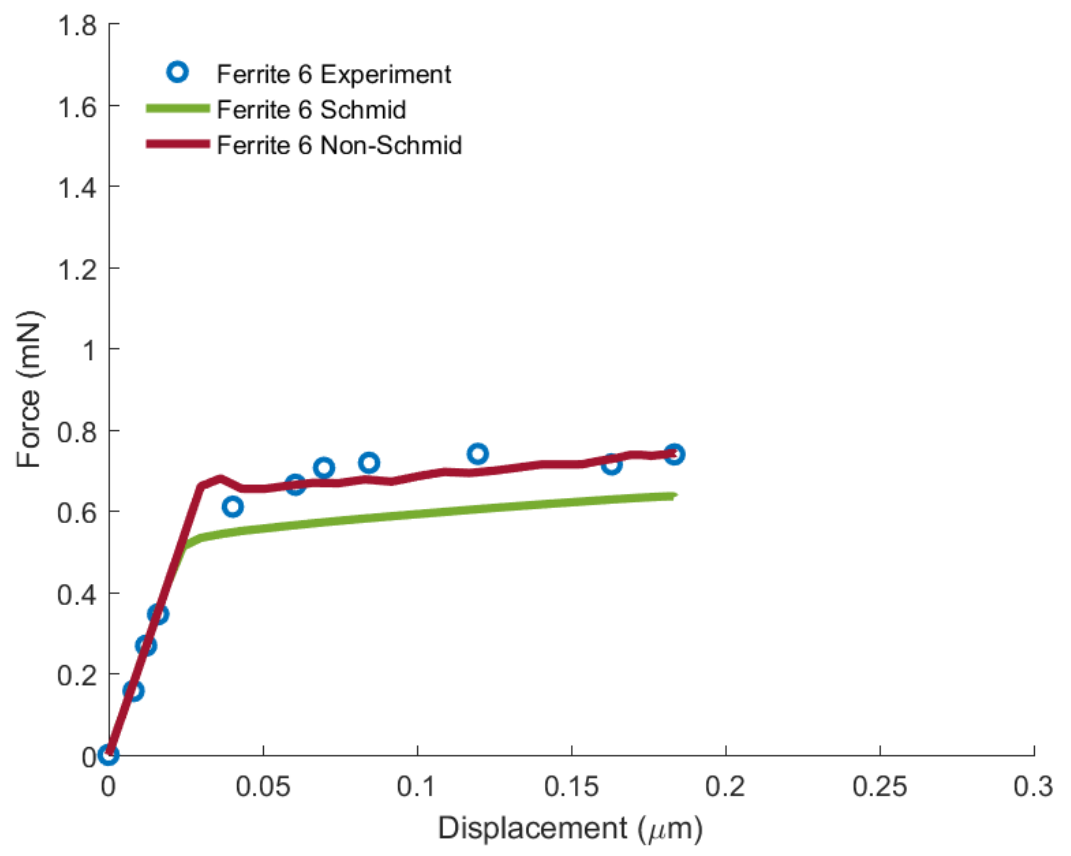

Figure 8: Comparing predictions of Schmid (green curve) and non-Schmid (red curve) models for ferrite micropillar 6 against the experiment. The Schmid model was simultaneously fitted to micropillars 1 and 4, while the non-Schmid model was calibrated with micropillars 1, 3 and 5. 
Figure 9: The contour shows the ratio of the non-Schmid resolved shear stress to the Schmid resolved shear stress equation 36 . Circles show the orientation of compression axis of ferrite micropillars 1-6. Green circles denote the orientations for which predictions of both the Schmid and non-Schmid models are accurate. Red circles show the orientations for which only the non-Schmid model gives good predictions. 
Figure 10: Comparing predictions of the Schmid model against the experiment when material parameters are calibrated using pairs of micropillars 1 and 6 (yellow curve) and micropillars 4 and 5 (purple curve). 


\section{CPFE modeling of uniaxial compression of DP980 ferrite micropil-} lar

Four single crystal ferrite micropillars were machined out of a dual-phase DP980 steel sheet using the same FIB technique that was discussed in the section. The samples were named A, B, C and D. These micropillars have a height to diameter ratio of $2-4$, a top surface diameter of about $1.3 \mu \mathrm{m}$ and a taper angle of $4^{\circ}$. Figure 2 shows a schematic of the cylindrical micropillar. Each micropillar was uniaxially compressed using a flat punch nano-indentor.

590 Figure 11 shows the orientation of these micropillars with respect to the loading axis. More details on the sample extraction and compression experiments can be found in 39 .

It is noted here that after the first few percent of plastic deformation (strains $<5 \%$ ), these micropillars exhibit large slip steps and often deviate from the uniaxial loading due to bending and twisting.

Unfortunately, the current test set-up and procedure do not lend themselves to interrupted testing or in-situ observations. Consequently, images of deformed micropillars were recorded at the end of each test using a scanning electron microscope (SEM). These images usually show significant micropillar distortion, as shown in Figure 14a.

\subsection{Modeling DP980 ferrite micropillars}

One of the most important parts of a crystal plasticity model is its hardening law, as it predicts the flow stress of each slip system. As mentioned before, the most commonly used hardening model is based on the pioneering work of Hill [35] and further development by authors like Hill and Rice [40, Havner and Baker [1, 42, Vause and Havner [43] and Asaro [36].

The main difference between these models is the definition of the hardening moduli matrix. The one defined by Asaro [36], equation (22), which is used in the simulation of QP980 micropillars only has two distinct parameters. One 
defines the self-hardening rate and the other defines the latent-hardening rate. This matrix, although convenient, only predicts good results until the end of stage I of deformation of a single crystal. Beyond this stage, the Asaro hardening moduli matrix is not accurate. The solid lines in Figures 12 and 13 compare the predictions of the non-Schmid crystal plasticity model with the conventional

${ }_{615}$ (Hill type) hardening rule and the Asaro type hardening moduli matrix for deformation of DP980 ferrite micropillars A and B against the experiments.

To improve the predictions of the Hill type hardening model, Madec and Kubin [44] studied the interactions of 12 slip systems of type $1 / 2\langle 111\rangle\{1 \overline{1} 0\}$ and 12 slip systems of type $1 / 2\langle 111\rangle\{11 \overline{2}\}$ and found 17 distinct interaction types out of 144 possible binary interactions. The hardening moduli matrix they defined, therefore, has 17 distinct coefficients. Researchers like [45, 47] used this matrix to model hardening. The dashed lines in Figures 12 and 13 compare the predictions of the non-Schmid model with the Hill type hardening rule and Madec-Kubin (MK) type hardening moduli matrix for deformation of DP980 ferrite micropillars A and B against the experiments. As can be seen in these figures, the predictions of the non-Schmid model with either of the hardening moduli are only accurate until the slope of the force-displacement curve changes and stage II begins.

Since the classical hardening model and moduli cannot accurately predict 630 the deformation of single crystals past stage I, the deformation of DP980 micropillars was modeled with the non-Schmid model with the DE hardening rule described in section 4.1. To ensure that FE simulation closely follows the complex deformation of the micropillar, care was taken to match the boundary conditions implemented in the CPFE model with those observed in the experiment. This allowed for qualitative comparison of the CPFE model results with the experimental curves beyond the uniform compression of the micropillar that was estimated to end around 5-6\% strain.

Figure 14 shows the actual deformed shape and the prediction of the nonSchmid model with DE hardening rule for micropillar A. Qualitatively, the predicted deformed shape of the micropillar is similar to the experiment. Due 
to the lack of a damage criterion, however, the CPFE model is not equipped to predict slip bands and discontinuities which result in the slip step observed in the experiment. The predicted compressive strain contour levels shown in Figure $14 \mathrm{~b}$ nonetheless, show similar shear bands forming in the same general

Uchic [48] explained one needs to use a gradient based model to consider the size effect. Kuroda [49] compared a conventional and a gradient based model to predict the deformation of an FCC material and argued that to accurately predict the deformation mode of micropillars one should use a gradient based deformation model. He also points out that there is no significant difference between the yield stress predictions of the conventional and gradient based models. The non-Schmid crystal plasticity model developed in this paper is not a gradient-based model. However, given Kuroda's [49] findings, this model should still be able to accurately predict the yield stress of the uniaxially compressed ferrite micropillar. Given that the primary purpose of this study was to accurately match the force-displacement response of micropillars for parameter calibration, the use of the conventional CPFE model is well justified.

As mentioned earlier, equation (31) due to its two adjustable parameters can only predict the hardening rate of the first stage of deformation. However, the new hardening model in equation (35) has three extra material constants, i.e. $k_{1}, k_{2}$ and $\nu$ that can be calibrated with an experimental force-displacement curve. In this study, to reduce the total number of calibration parameters and increase the calibration speed, the non-Schmid parameters for DP980 ferrite micropillars, i.e. $a_{1}, a_{2}$ and $a_{3}$, were taken from the literature [26].

Commercial optimization software LS-OPT ${ }^{\circledR}$ was used to calibrate the model the compression test of ferrite micropillars A and B. Table 5 shows the material parameters found from this calibration. Figures 15 and 16 show the experimental and predicted force-displacement curves for these micropillars, which are in very good agreement.

Next, the compression response of the other two ferrite micropillars, i.e. C and $\mathrm{D}$, were predicted with the calibrated non-Schmid crystal plasticity model 
using the Differential-Exponential (DE) hardening rule. Figures 17 and 18 compare predictions of the CPFE model with remaining two experiments (i.e. C and D) using the same hardening parameters shown in Table 5 As can be seen in these figures, predictions of the DE hardening model are in good agreement with the force-displacement curves of the DP980 ferrite micropillars. This confirms the validity of the DE hardening model, and as a result the corresponding criteria that were used to define the initiation of the stage II hardening. The black stars in figures 15 through 17 indicate the point at which the DE hardening model switched the hardening rule from stage I to stage II. Figure 18 shows that for micropillar D, stage I hardening is dominant and stage II hardening never starts.

To study the mesh sensitivity of the DE hardening model, simulations for all micropillars were repeated with finer meshes. These are also shown in Figures 68515 through 18 with a dashed line. As can be seen in these figures, the model is not mesh sensitive. Number of elements in each case are shown in Table 6 .

As was discussed earlier, the second stage hardening commences when secondary slip systems become active and interact with the primary slip system. The activation of secondary slip systems is mainly caused by the crystal rotation which makes these systems more favorable, as well as the increase in the resolved shear stress beyond the CRSS value for secondary slip systems. This justifies the use of $\max \left(\tau^{\beta}\right)$ as one of the criteria. Figure 19 shows the evolution of $\frac{\max \left(\tau^{\beta}\right)}{\tau_{s}}$ parameter with the increasing engineering strain. The critical value $k_{1}$ is shown by a dashed black line. It is interesting to note that micropillars with different initial orientations reach the critical value at different engineering strain levels. It should be noted from Figure 18 that unlike micropillars A, B and $\mathrm{C}$, micropillar $\mathrm{D}$ never reaches the critical value for $k_{1}$.

The maximum slip resistance among all slip systems, however, cannot independently signal the onset of stage II hardening. Additionally, dislocations on secondary slip systems must start interacting and intersecting dislocations on the primary system. These dislocation interactions can act as barriers, thus increase the slip resistance and harden the material. Therefore, the ratio $\frac{\gamma^{2 n d}}{\gamma^{1 s t}}$ was 


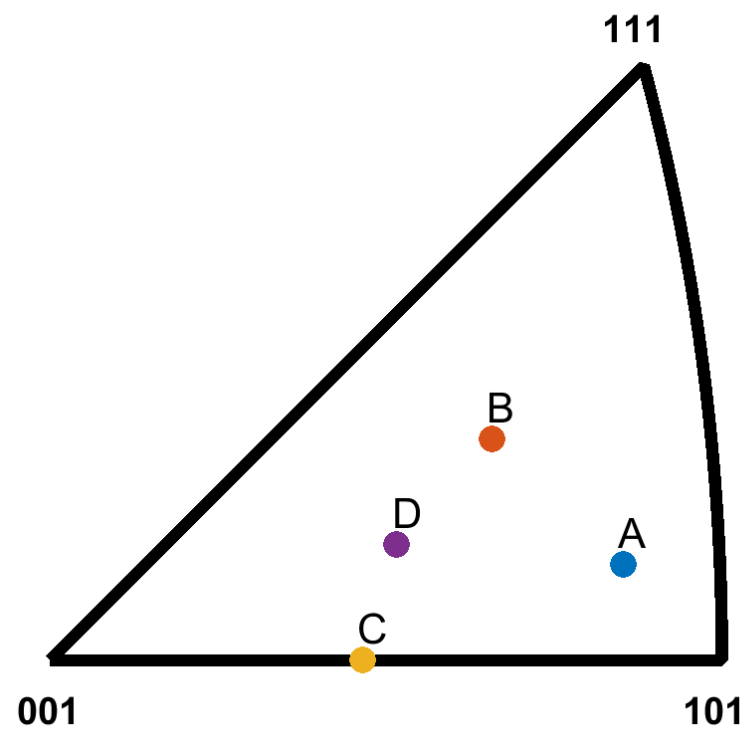

Figure 11: This inverse pole figure shows the orientation of compression axis of the DP980 ferrite micropillars A-D.

also chosen as part of the criteria for the initiation of stage II hardening. Figure 20 shows the evolution of this criterion and the dashed black line represents the critical value. Figures 19 and 20 clearly show that these two conditions are met at different engineering strains for pillars A, B, C, and D. In other words, the stage II hardening occurs dynamically and is dependent on the loading direction and the crystal orientation.

Table 5: Material parameters found for the non-Schmid modeling of DP980 (calibrated to micropillars A and B).

\begin{tabular}{|c|c|c|c|c|c|c|c|c|c|c|}
\hline$\tau_{S}$ & $\tau_{0}$ & $h_{0}$ & $h_{0 D E}$ & $\eta$ & $k_{1}$ & $k_{2}$ & $\nu$ & $a_{1}$ & $a_{2}$ & $a_{3}$ \\
\hline 840 & 190 & 200 & 44060 & 3 & 0.2538 & 0.0700 & 3 & 0.0293 & 0.1727 & 0.3000 \\
\hline
\end{tabular}




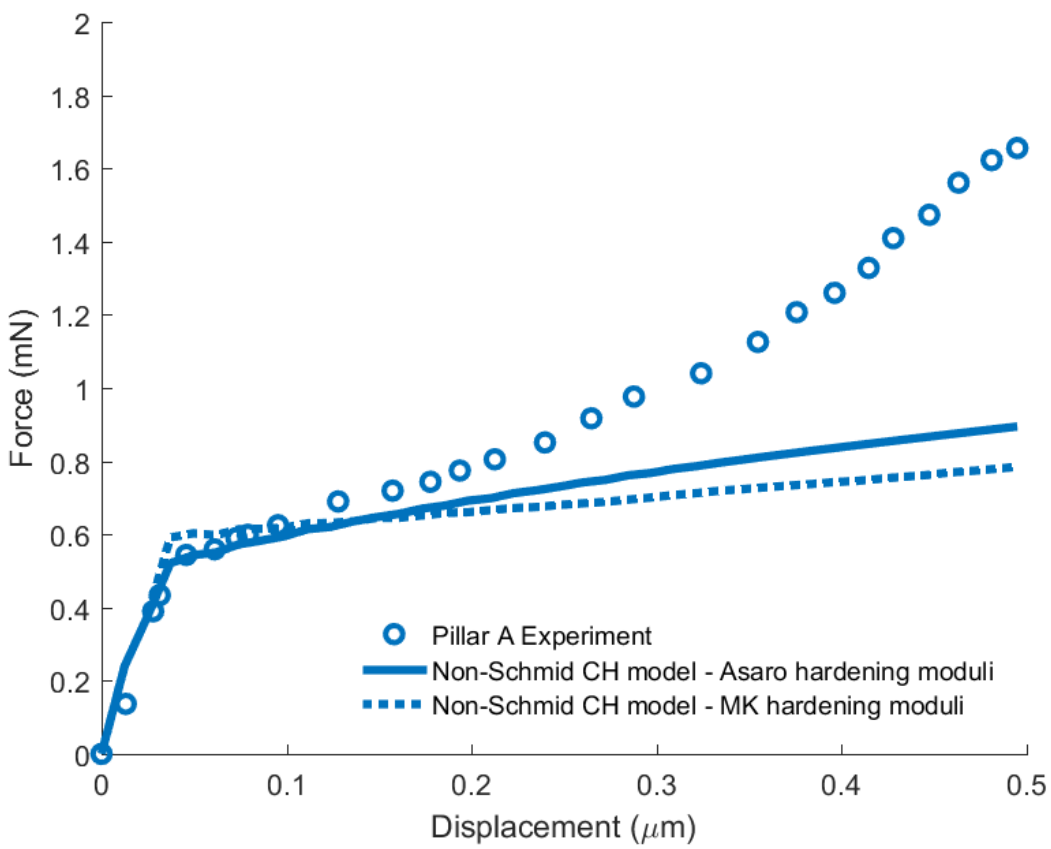

Figure 12: Comparing predictions of the non-Schmid model with classical hardening rule and two hardening moduli against the experiment of DP980 ferrite micropillar A. The circles show the experimental data, the solid line shows the predictions of the model with the Asaro hardening moduli and the dashed line represents the predictions of the model with the MadecKubin (MK) hardening moduli. As can be seen, the predictions are close to the experiment in the stage I of deformation before the rate of hardening changes.

Table 6: Number of elements in coarse and fine mesh cases for DP980 ferrite micropillars.

\begin{tabular}{|c|c|c|}
\hline \multirow{2}{*}{ Micropillar } & \multicolumn{2}{|c|}{ Number of Elements in } \\
\cline { 2 - 3 } & Coarse Mesh & Fine Mesh \\
\hline A & 640 & 3276 \\
\hline B & 640 & 3040 \\
\hline C & 480 & 3217 \\
\hline D & 480 & 3840 \\
\hline
\end{tabular}




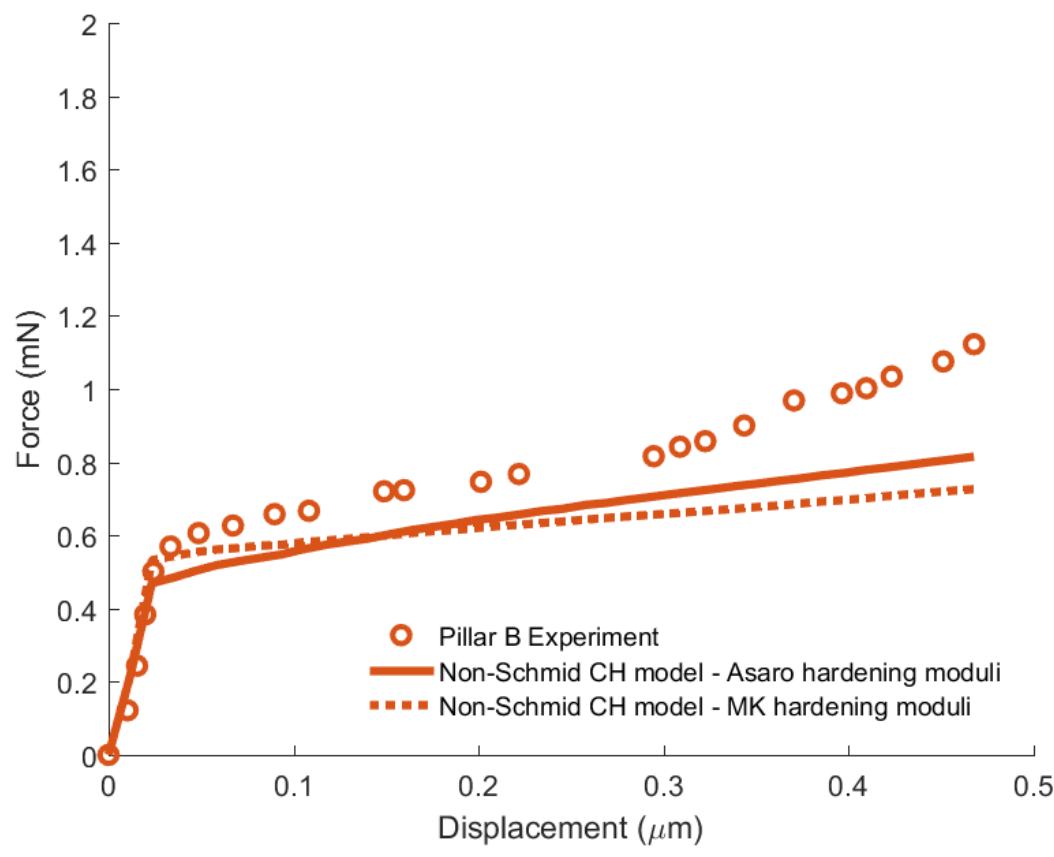

Figure 13: Comparing predictions of the non-Schmid model with classical hardening rule and two hardening moduli against the experiment of DP980 ferrite micropillar B. The solid line represents the predictions of the model with the Asaro hardening moduli and the dashed line shows the predictions of the model with the Madec-Kubin (MK) hardening moduli. The circles show the experimental data. 


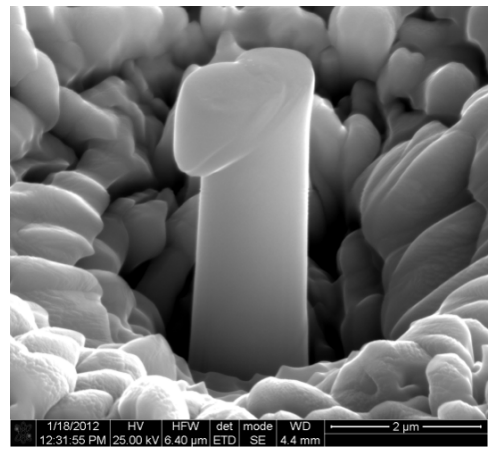

(a)

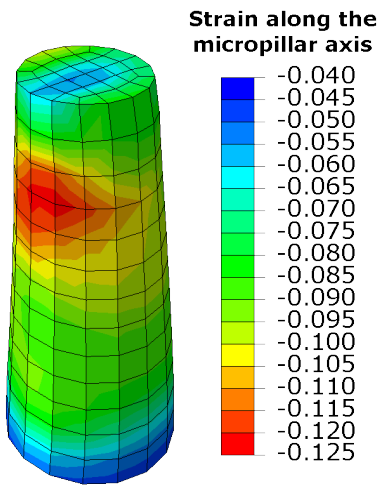

(b)

Figure 14: This figure compares the geometry of the deformed ferrite A from (a) experiment with the (b) prediction of the non-Schmid model with DE hardening rule. The contour shows the compressive strain parallel to the axis of the micropillar. The current crystal plasticity model does not have a damage model. Therefore, it cannot show the slip steps. The contour levels, however, qualitatively match with the slip step observed in the experiment.

\section{Conclusions}

According to the Schmid law, only shear stresses resolved on the slip plane and parallel to the slip direction affect the dislocation motion. Although this is true for FCC materials, it does not represent the more complex deformation behavior of BCC materials. In these materials, other stresses that are not parallel and/or non-planar to the slip direction may also affect the dislocation motion.

In this study, the uniaxial compression of single crystal ferrite micropillars of QP980 steel were simulated with Schmid and non-Schmid crystal plasticity models, and their predicted force-displacement curves were compared with experimental curves. For some special orientations, predictions of both models were identical. Generally, however, only predictions of the non-Schmid type crystal plasticity model accurately matched the experimental force-displacement curves of ferrite micropillars.

The Schmid model consistently overestimated the force-displacement of mi- 


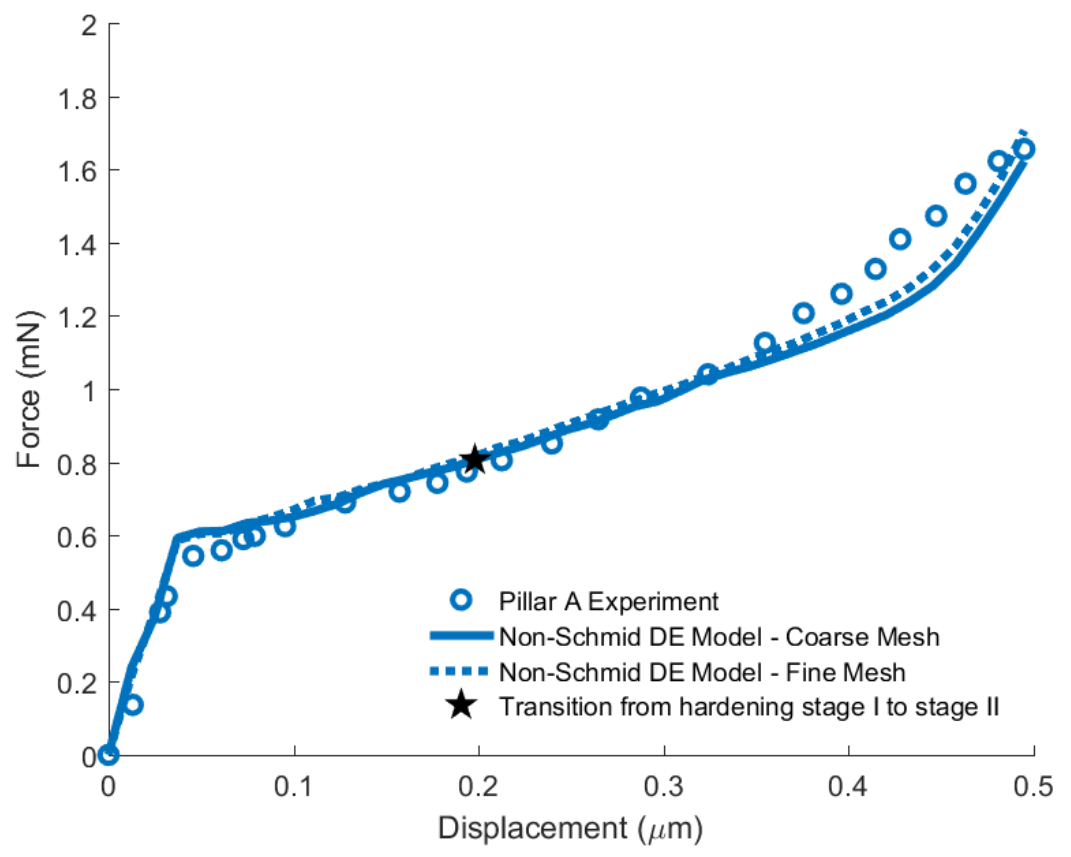

Figure 15: The Differential-Exponential non-Schmid crystal plasticity model was calibrated using DP980 single crystal ferrite micropillars A and B. The solid line shows the calibration of the model (with coarse mesh) against the experiment of DP980 ferrite micropillar A. The dashed line is the predictions of the model with a finer mesh. The black star represents the point at which the hardening rule switches from the stage I to stage II. 


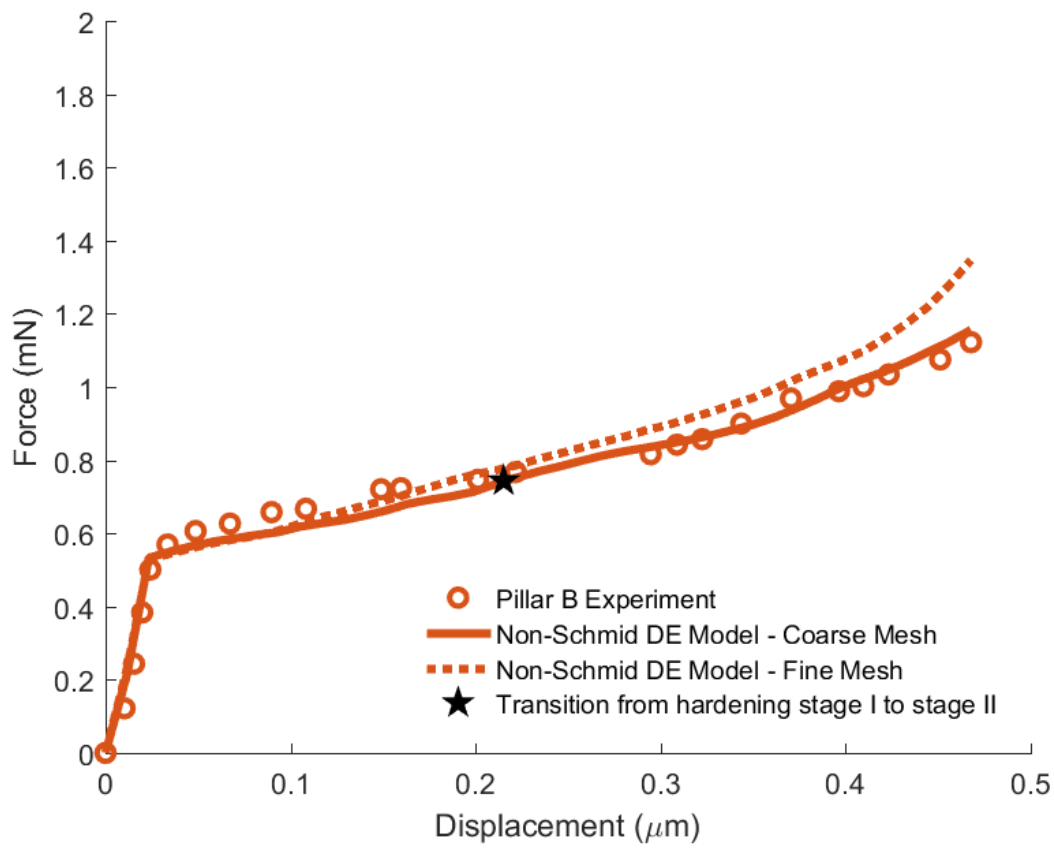

Figure 16: The Differential-Exponential non-Schmid crystal plasticity model was calibrated using DP980 single crystal ferrite micropillars A and B. The solid line shows the calibration of the model (with coarse mesh) against the experiment of ferrite micropillar B. The dashed line is the predictions of the model with a finer mesh. The black star represents the point at which the hardening rule switches from the stage I to stage II. 


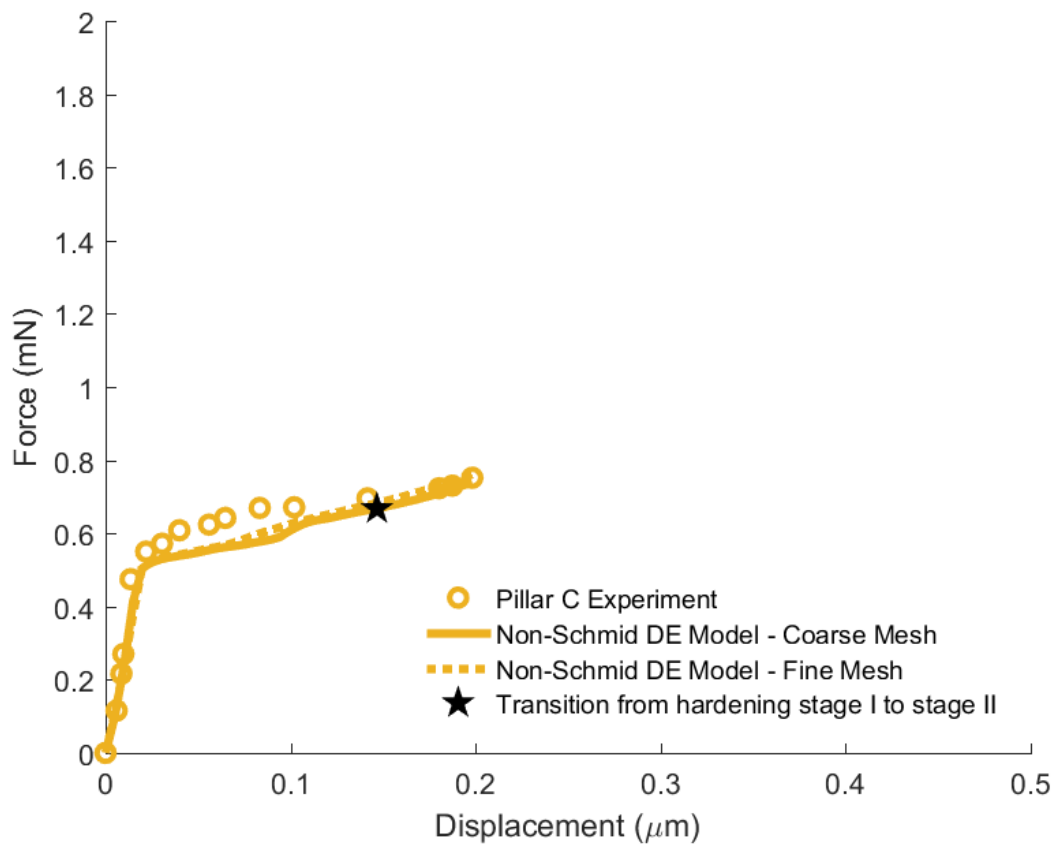

Figure 17: Comparing predictions of the Differential-Exponential non-Schmid crystal plasticity model for DP980 single crystal ferrite micropillar C against the experiment. The solid line shows the predicted behavior with the coarse mesh and the dashed line shows the predicted behavior with the fine mesh. The model is not mesh sensitive. The black star represents the point at which the hardening rule switches from the stage I to stage II. 


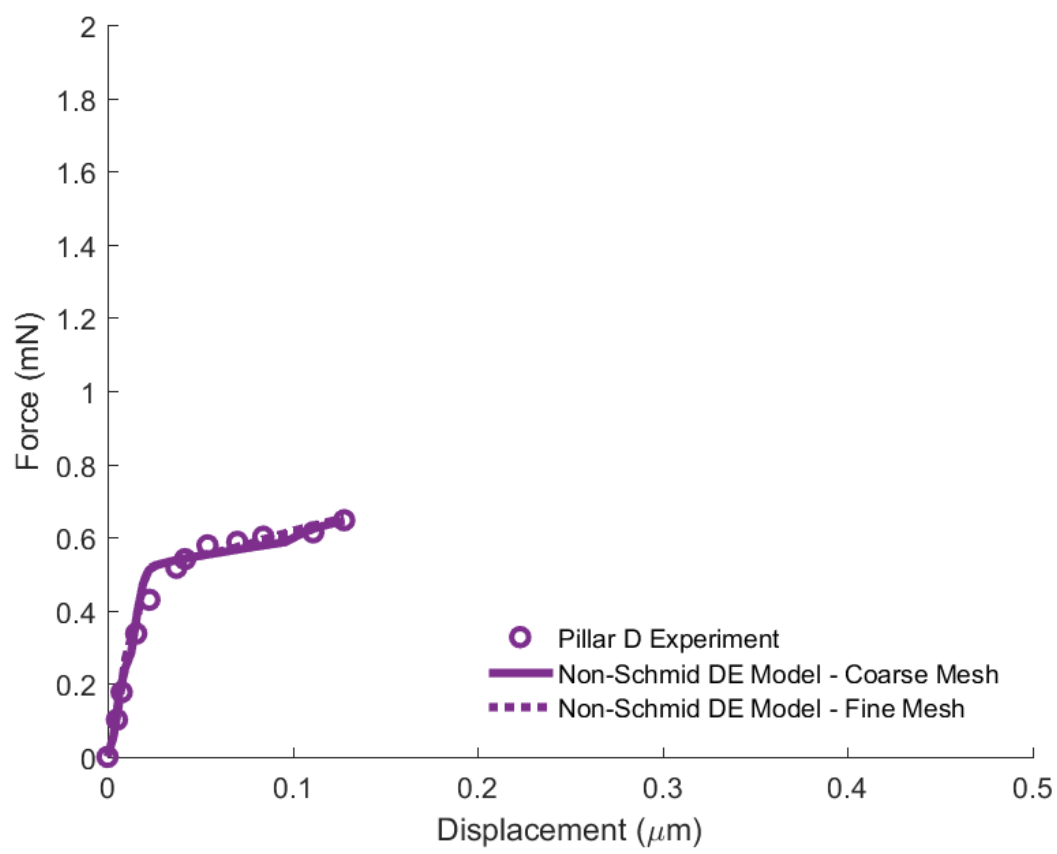

Figure 18: Comparing predictions of the Differential-Exponential non-Schmid crystal plasticity model for DP980 single crystal ferrite micropillar D against the experiment. The solid line shows the predicted behavior with the coarse mesh and the dashed line shows the predicted behavior with the fine mesh. The model is not mesh sensitive. 


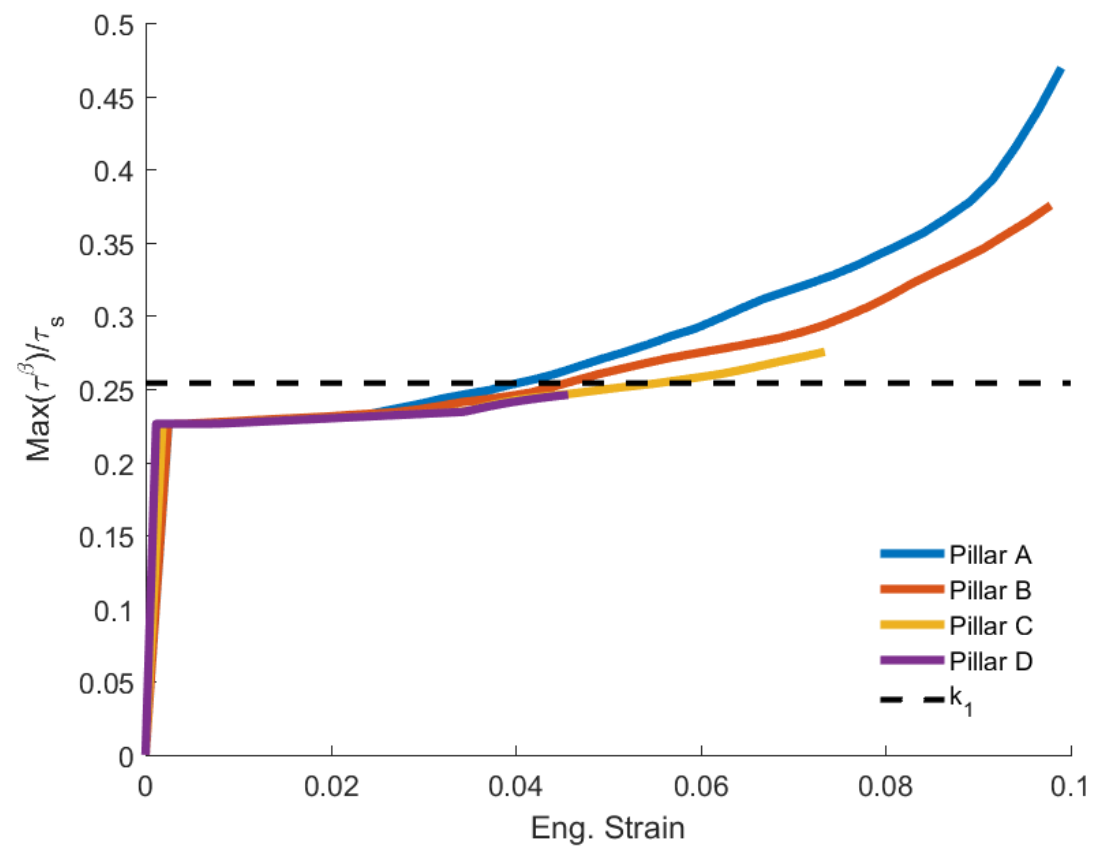

Figure 19: This plot shows the evolution of $\frac{\max \left(\tau^{\beta}\right)}{\tau_{s}}$ for each (coarse mesh) micropillar during the deformation. One of the conditions of the DE hardening model is met when this parameter reaches $k_{1}$, which is shown with the dashed black line. 


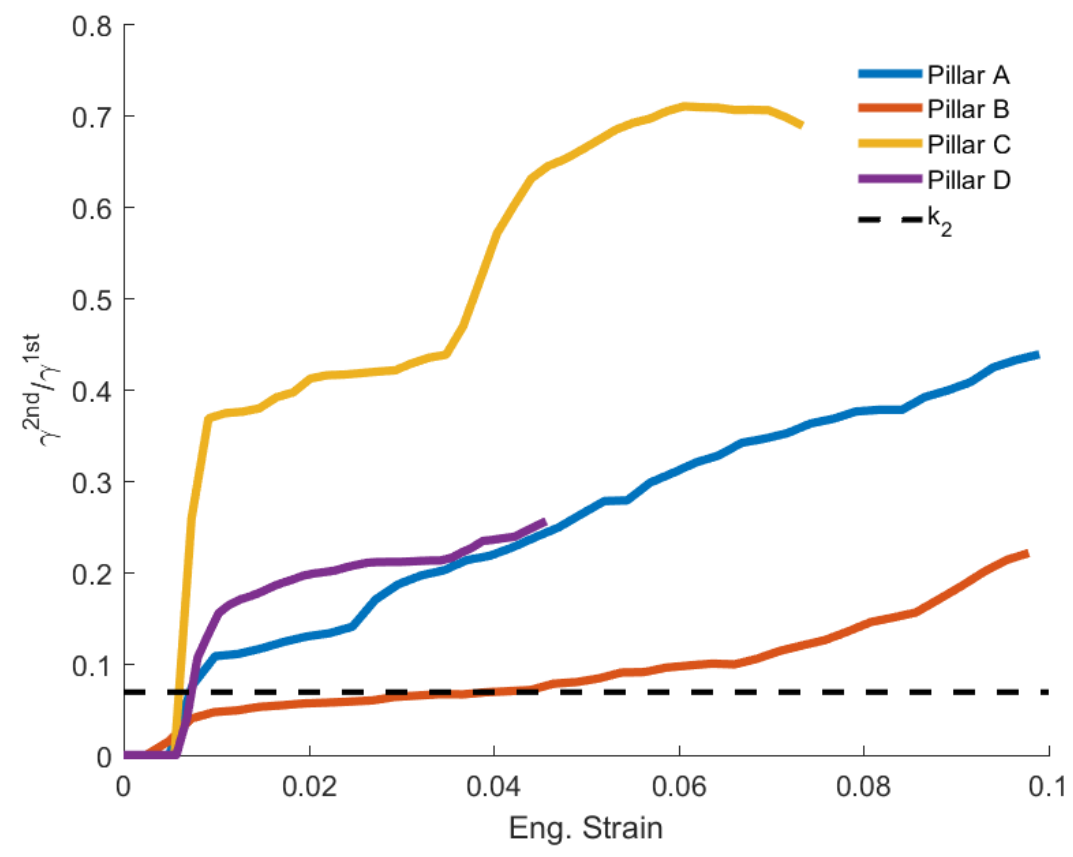

Figure 20: This plot shows the evolution of $\frac{\gamma^{2 n d}}{\gamma^{1 s t}}$ for each (coarse mesh) micropillar during the deformation. One of the conditions of the DE hardening model is met when this parameter reaches $k_{2}$, which is shown with the dashed black line. 
cropillars with orientations that had $R_{n s}$ values greater than those used for the calibration, and underestimated the force-displacement curves of those micropillars that had smaller $R_{n s}$ values.

The classical Hill's power-law hardening used in crystal plasticity models can only predict the stage I hardening. In this study, an exponential hardening model was proposed to also model the stage II hardening. The accuracy of this model, termed as Differential-Exponential (DE) hardening, was verified against the uniaxial compression of single crystal ferrite micropillars of DP980 steel. It was shown that the DE hardening model can accurately predict stage I and stage II hardening in these single crystals.

Another important finding of this study was that calibrating the material 735 parameters of the non-Schmid CPFE model using only one micropillar compression force-displacement curve will not be sufficient, and will result in invalid material parameters. This study shows that calibrating with two independent experimental force-displacement curves significantly improves the consistency of fitted parameters, and also increases the accuracy of the model's predictions.

\section{Acknowledgments}

This material is based upon work supported by the Department of Energy under Cooperative Agreement Number DOE DE-EE000597, with United States Automotive Materials Partnership LLC (USAMP).

This report was prepared as an account of work sponsored by an agency of the United States Government. Neither the United States Government nor any agency thereof, nor any of their employees, makes any warranty, express or implied, or assumes any legal liability or responsibility for the accuracy, completeness, or usefulness of any information, apparatus, product, or process disclosed, or represents that its use would not infringe privately owned rights. Reference herein to any specific commercial product, process, or service by trade name, trademark, manufacturer, or otherwise does not necessarily constitute or imply its endorsement, recommendation, or favoring by the United States 
Government or any agency thereof. The views and opinions of authors expressed herein do not necessarily state or reflect those of the United States Government or any agency thereof.

\section{References}

n [1] Z. Wang, I. Beyerlein, An atomistically-informed dislocation dynamics

a model for the plastic anisotropy and tensioncompression asymmetry of BCC metals, International Journal of Plasticity 27 (2011) 1471-1484.

口 URL http://linkinghub.elsevier.com/retrieve/pii/ S0749641910001166

[ [2] M. Knezevic, D. J. Savage, A high-performance computational framework for fast crystal plasticity simulations, Computational Materials Science 83 (2014) 101-106. doi:10.1016/j.commatsci.2013.11.012

URL http://linkinghub.elsevier.com/retrieve/pii/ S0927025613006848

[3] A. Mapar, T. R. Bieler, F. Pourboghrat, C. Compton, Dynamic hardening rule; a generalization of the classical hardening rule for crystal plasticity, in: 16th International Conference on RF Superconductivity, Paris, France, 2013, pp. 499-503.

[4] A. Mapar, T. R. Bieler, F. Pourboghrat, C. C. Compton, Crystal Plasticity Finite Element Modeling of Single Crystal Niobium Tensile Tests with Weighted Dynamic Hardening Rule, in: M. Li, C. Campbell, K. Thornton, E. Holm, P. Gumbsch (Eds.), 2nd World Congress on Integrated Computational Materials Engineering, TMS (The Minerals, Metals \& Materials Society), 2013, pp. 255-258.

[5] A. Mapar, D. Kang, T. R. Bieler, F. Pourboghrat, C. C. Compton, Crystal plasticity modeling of single crystal niobium, in: R. E. Laxdal, J. Thomson, 
V. R. Schaa (Eds.), 17th International Conference on RF Superconductivity, JACoW, Geneva, Switzerland, Whistler, British Columbia, Canada, 2015, pp. 228-232. doi:10.18429/JACoW-SRF2015-MOPB057

[6] V. Vítek, R. C. Perrin, D. K. Bowen, The core structure of (111) screw dislocations in B.C.C. crystals, Philosophical Magazine 21 (173) (1970)

[ [7] M. S. Duesbery, On Non-Glide Stresses and Their Influence on the Screw Dislocation Core in Body-Centred Cubic Metals. I. The Peierls Stress, Proceedings of the Royal Society A: Mathematical, Physical and Engineering Sciences 392 (1802) (1984) 145-173. doi:10.1098/rspa.1984.0027

[ [8] M. S. Duesbery, On Non-Glide Stresses and Their Influence on the Screw Dislocation Core in Body-Centred Cubic Metals II. The Core Structure, Proceedings of the Royal Society A: Mathematical, Physical and Engineer-

[11] A. Koester, A. Ma, A. Hartmaier, Atomistically informed crystal plasticity model for body-centered cubic iron, Acta Materialia 60 (9) (2012) 3894-3901. doi:10.1016/j.actamat.2012.03.053

805 
[12] J. Bassani, K. Ito, V. Vitek, Complex macroscopic plastic flow arising from non-planar dislocation core structures, Materials Science and Engineering: A 319-321 (2001) 97-101. doi:10.1016/S0921-5093(00)02008-6. S0921509300020086

[13] V. Vitek, M. Mrovec, R. Gröger, J. Bassani, V. Racherla, L. Yin, Effects

1 of non-glide stresses on the plastic flow of single and polycrystals of molybdenum, Materials Science and Engineering: A 387-389 (2004) 138-142. doi:10.1016/j.msea.2004.04.066

口 URL http://linkinghub.elsevier.com/retrieve/pii/ S0921509304005301

[14] R. Gröger, V. Vitek, Breakdown of the Schmid Law in BCC Molybdenum Related to the Effect of Shear Stress Perpendicular to the Slip Direction, Materials Science Forum 482 (2005) 123-126. doi:10.4028/www. scientific.net/MSF.482.123.

URL http://www.scientific.net/MSF . 482.123

[15] R. Gröger, V. Vitek, Multiscale modeling of plastic deformaa tion of molybdenum and tungsten. III. Effects of temperature 825 and plastic strain rate, Acta Materialia 56 (19) (2008) 5426-5439. doi:10.1016/j.actamat.2008.07.027.

10 URL http://linkinghub.elsevier.com/retrieve/pii/ S1359645408005156

[16] R. Gröger, A. Bailey, V. Vitek, Multiscale modeling of plastic deformation ${ }_{830}$ of molybdenum and tungsten: I. Atomistic studies of the core structure and glide of $1 / 2111$ screw dislocations at 0K, Acta Materialia 56 (19) (2008) 5401-5411. doi:10.1016/j.actamat.2008.07.018.

प URL http://linkinghub.elsevier.com/retrieve/pii/ S1359645408005132 
835 [17] R. Gröger, V. Racherla, J. Bassani, V. Vitek, Multiscale model-

n ing of plastic deformation of molybdenum and tungsten: II. Yield

a criterion for single crystals based on atomistic studies of glide of $1 / 2111$ screw dislocations, Acta Materialia 56 (19) (2008) 5412-5425. doi:10.1016/j.actamat.2008.07.037.

${ }_{840}$ ॥ URL http://linkinghub.elsevier.com/retrieve/pii/

\section{S1359645408005144}

[18] T. E. Buchheit, C. C. Battaile, C. R. Weinberger, E. A. Holm, Multi-scale modeling of low-temperature deformation in b.c.c. metals, JOM 63 (11) (2011) 33-36. doi:10.1007/s11837-011-0188-x.

845

URL

http://www.springerlink.com/index/10.1007/ s11837-011-0188-x

[19] C. R. Weinberger, C. C. Battaile, T. E. Buchheit, E. a. Holm, In1 corporating atomistic data of lattice friction into BCC crystal plasticity models, International Journal of Plasticity 37 (2012) 16-30.

850 doi:10.1016/j.ijplas.2012.03.012.

a

URL http://linkinghub.elsevier.com/retrieve/pii/ S0749641912000526

[20] K. Srivastava, R. Gröger, D. Weygand, P. Gumbsch, Dislocation a motion in tungsten: Atomistic input to discrete dislocation sim855 ulations, International Journal of Plasticity 47 (0) (2013) 1-17. doi:10.1016/j.ijplas.2013.01.014.

a URL http://linkinghub.elsevier.com/retrieve/pii/

S0749641913000247\$delimiter"026E30F\$nhttp://www. sciencedirect.com/science/article/pii/S0749641913000247

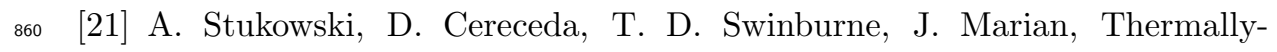
activated non-Schmid glide of screw dislocations in W using atomisticallyinformed kinetic Monte Carlo simulations, International Journal of Plasticity 65 (2015) 108-130. doi:10.1016/j.ijplas.2014.08.015. 
[22] D. Cereceda, M. Diehl, F. Roters, D. Raabe, J. M. Perlado, J. Marian, Un-

[23] M. Knezevic, I. J. Beyerlein, M. L. Lovato, C. N. Tomé, A. W. Richards, response of dual-phase steels, Acta Materialia 65 (2014) 133-149. doi:10.1016/j.actamat.2013.11.036. 890

[26] A. Patra, T. Zhu, D. L. McDowell, Constitutive equations for moda eling non-Schmid effects in single crystal bcc-Fe at low and ambient 
temperatures, International Journal of Plasticity 59 (2014) 1-14.

[27] A. Srivastava, H. Ghassemi-Armaki, H. Sung, P. Chen, S. Kumar, A. F. Bower, Micromechanics of plastic deformation and phase transformation

1 in a three-phase TRIP-assisted advanced high strength steel: Experiments and modeling, Journal of the Mechanics and Physics of Solids 78 (2015) 46-69. doi:10.1016/j.jmps.2015.01.014.

URL

http://linkinghub.elsevier.com/retrieve/pii/ S0022509615000290

[28] H. Lim, L. M. Hale, J. a. Zimmerman, C. C. Battaile, C. R. Weinberger, A multi-scale model of dislocation plasticity in $\alpha$-Fe: Incorporating temperature, strain rate and non-Schmid effects, International Journal of Plasticity 73 (2015) 100-118. doi:10.1016/j.ijplas.2014.12.005.

口 URL http://ww.sciencedirect.com/science/article/pii/ S0749641914002356

910

[30] A. Zamiri, F. Pourboghrat, F. Barlat, An effective computational algo915

口

920 घ of tube hydroforming of polycrystalline aluminum alloy extru- 
sions, International Journal of Plasticity 22 (12) (2006) 2366-2393. doi:10.1016/j.ijplas.2006.04.003

925

[32] D. Hull, D. J. Bacon, Introduction to Dislocations, 3rd Edition, Oxford [Oxfordshire]: Pergamon Press, 1984.

[33] A. R. Zamiri, F. Pourboghrat, A novel yield function for single crystals based on combined constraints optimization, International Journal of Plasticity 26 (5) (2010) 731-746. doi:10.1016/j.ijplas.2009.10.004

प URL http://linkinghub.elsevier.com/retrieve/pii/ S0749641909001284

[34] J. Bassani, V. Racherla, From non-planar dislocation cores to nonassociated plasticity and strain bursts, Progress in Materials Science 56 (6)

[37] J. J. Gilman, Micromechanics of flow in solids, McGraw-Hill, New York, 1969.

[38] N. Stander, A. Basudhar, U. Basu, I. Gandikota, V. Savic, X. Sun, K. S. Armaki, F. Abu-Farha, Multi-scale Material Parameter Identification Using LS-DYNA $\AA$ and LS-OPT $\AA$, in: 10th European LS-DYNA Conference, DYNAmore GmbH, Würzburg, Germany, 2015. 
[39] H. Ghassemi-Armaki, R. Maaß, S. P. Bhat, S. Sriram, J. R. Greer, K. S.

प 2013.10.001.

URL http://dx.doi.org/10.1016/j.actamat.2013.10.001

[40] R. Hill, J. Rice, Constitutive analysis of elastic-plastic crystals at arbitrary

[45] S. Queyreau, G. Monnet, B. Devincre, Slip systems interactions in $\alpha$-iron determined by dislocation dynamics simulations, International Journal of Plasticity 25 (2) (2009) 361-377. doi:10.1016/j.ijplas.2007.12.009

[41] K. S. Havner, G. S. Baker, Theoretical latent hardening in crystals - I BCC crystals in tension and compression, Journal of the Mechanics and Physics of Solids 27 (4) (1979) 285-314. doi:10.1016/0022-5096(79)90031-0.

[42] K. S. Havner, G. S. Baker, Theoretical latent hardening in crystals - II BCC crystals in tension and compression, Journal of the Mechanics and Physics of Solids 27 (4) (1979) 285-314. doi:10.1016/0022-5096(79)90031-0.

[43] R. F. Vause, K. S. Havner, Theoretical latent hardening in Crystals - III FCC Crystals in Compression, J. Mech. Phys. Solids 27 (1979) 393-414.

[44] R. Madec, L. P. Kubin, Dislocation Interactions and Symmetries in BCC

घ Crystals, Springer Netherlands, Dordrecht, 2004, pp. 69-78. doi:10.1007/ 978-1-4020-2111-4_7.

URL http://dx.doi.org/10.1007/978-1-4020-2111-4\{_\}7

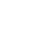

401-413. doi:10.1016/0022-5096(72)90017-8.

【 URL http://linkinghub.elsevier.com/retrieve/pii/ 0022509672900178 
[46] B. Liu, D. Raabe, P. Eisenlohr, F. Roters, A. Arsenlis, G. Hommes, Dislocation interactions and low-angle grain boundary strengthening, Acta Materialia 59 (19) (2011) 7125-7134. doi:10.1016/j.actamat.2011.07.067 URL http://dx.doi.org/10.1016/j.actamat.2011.07.067

[47] C. Gérard, G. Cailletaud, B. Bacroix, Modeling of latent hardening produced by complex loading paths in FCC alloys, International Journal of Plasticity 42 (2013) 194-212. doi:10.1016/j.ijplas.2012.10.010.

[48] M. D. Uchic, Sample Dimensions Influence Strength and Crystal Plasticity Science 305 (5686) (2004) 986-989. doi:10.1126/science.1098993.

[49] M. Kuroda, Higher-order gradient effects in micropillar compression, Acta Materialia 61 (7) (2013) 2283-2297. doi:10.1016/j.actamat.2012.12. 038.

URL http://dx.doi.org/10.1016/j.actamat.2012.12.038

[50] N. Stander, W. Roux, A. Basudhar, T. Eggleston, T. Goel, K. Craig, LSOPT @ User's Manual - A Design Optimization and Probabilistic Analysis Tool, version 5. Edition, no. December, Livermore Software Technology Corporation, 2015.

\section{Appendix}

\section{A. Crystal plasticity finite element model calibration method}

To start, microscale experiments were conducted where the Focused Ion Beam (FIB) was used to carve out single crystal ferrite micropillars with different initial orientations from the ferrite phase of a three-phase quenched and partitioned QP980 commercial steel sheet. These micropillars were uniaxially compressed with a flat punch nano-indenter to determine their force-displacement 
response. It was found that beyond a certain displacement, micropillars were no longer deforming under uniaxial compression, rather they were experiencing a three-dimensional deformation. This was an important observation, since it became obvious that the micropillar compression test has to be modeled 
In this equation $N$ is the number of experimental measurements, $g_{n}(x)$ (where $n=1 \ldots N)$ is the value of each measurement, $f_{n}(u, x)$ is the corresponding predicted value and $u$ is the vector of design parameters (in this case material parameters). $g_{n}(x)$ is independent of this vector. $x$ is an independent state variable and depending on the problem can represent time, strain or any other type of response. In this study $x$, represents the displacement. The meansquare error is then normalized by $\max \left|g_{n}(x)\right|$ which is the maximum of the absolute value of $g_{n}(x)$. The result $e$ is a dimensionless scalar of order unity.

LS-OPT ${ }^{\circledR}$ was used to loop through the iterative process of running simulations, extracting the force-displacement, finding the mean-square error and refining input parameters. To do so a user-defined script was developed to run the simulations and extract the force-displacement data. In each iteration, depending on the size of the problem (number of parameters to optimize), a number of designs were evaluated. LS-OPT ${ }^{\circledR}$ then compared the NMSE for all designs within the iteration, chose the best design, made a new set of designs for the next iteration and started the simulations over. The termination criteria were either reaching a maximum number of iterations or when the difference between the two consecutive designs was less than a tolerance and the change in the two consecutive objective functions was less than another tolerance [50].

The NMSE gives the square of error. The metric used in this paper to quantify the quality of calibrations is the signed normalized root-mean-square error (S-NRMSE) which is defined by the following equation:

$$
e_{s}=\operatorname{sgn}\left(\frac{1}{N} \sum_{n=1}^{N}\left[f_{n}(u, x)-g_{n}(x)\right]\right) \sqrt{\frac{1}{N} \sum_{n=1}^{N}\left[\frac{f_{n}(u, x)-g_{n}(x)}{\max \left|g_{n}(x)\right|}\right]^{2}} \times 100
$$

The term in the sign function is the average error between the model prediction and the experiment. A positive value means the model overestimates the force, while a negative value implies the model underestimates the force. The term inside the square root is the NMSE as defined by equation A.1. Taking the square root and multiplying it by 100 gives the percentage error for 
each prediction and makes the results easier to understand. When the signs are neglected, results of equations A.1 and A.2 are consistent

In the non-Schmid model, there are seven material parameters $\left(\tau_{S}, \tau_{0}, h_{0}\right.$, $\eta, a_{1}, a_{2}$ and $\left.a_{3}\right)$ that can be fitted. In this study, $h_{0}=10$ and $\eta=3$ were kept constants and the remaining five parameters were found from the calibrations.

At first, the non-Schmid CPFE model was calibrated with only one micropillar at a time. The material parameters for each individual calibration is shown in Table A.1. Table A.2 shows the matrix of S-NRMSE values calculated based on this calibration. For example, the value of 37.68 appearing at the intersection of row 3 and column 1 implies that there was a $37.68 \%$ error when the CPFE model calibrated with micropillar 1 was used to predict the forcedisplacement of micropillar 3. The positive sign of S-NRMSE implies that the predicted force-displacement curve overestimated the experimental curve (see Figure A.2.

Figure A.1 shows the non-Schmid model calibrated to QP980 ferrite micropillar 1. Although the model closely predicts the behavior of micropillar 1, Table A.2 shows that its prediction for micropillars 3,5 and 6 are inaccurate. Figure A.2 shows the predictions of the non-Schmid CPFE model for QP980 ferrite micropillars 3 and 4 when the model is calibrated with micropillar 1 . As can be seen in Table A.2, the same model has the largest S-NRMSE for micropillar 3 (i.e. 37.68\%), and the second smallest S-NRMSE for micropillar 4 (i.e. $8.36 \%)$.

The last row of Table A.2 shows the sum of absolute error (SAE) for each calibration. This sum was used as a criterion to compare the quality of calibrations. According to Table A.2, calibration of the non-Schmid CPFE model using micropillar 4 results in the best total performance, since it has the smallest SAE of 26.95. While using only one micropillar to calibrate the CPFE model might seem ideal, according to Table A.2, only 1 out of 6 calibrations resulted in accurate prediction of other force-displacement curves, which is statistically insignificant.

Given the low rate of success when using just one micropillar, it was decided 
to calibrate the non-Schmid CPFE model with two and three micropillars. Table A.3 shows the material parameters found for a select number of calibrations using combinations of 2 and 3 micropillar force-displacement curves. Table A.4 shows the matrix of S-NRMSE values when the calibrated non-Schmid CPFE model was used to predict the force-displacement curves of the remaining micropillars. By inspecting Table A.4 it is apparent that calibrations with 2 and 3 micropillars consistently produce small errors, with the smallest error being for micropillar 2, and the largest error being for micropillar 5. Figure A.3 shows the result of the non-Schmid CPFE model simultaneously calibrated to QP980 ferrite micropillar 1 and 4. Figure A.4 shows the force-displacement predictions of the same calibrated non-Schmid CPFE model for micropillars 2 (best) and 5 (worse).

Figure A.5 shows the calibration of the non-Schmid CPFE model with several distinct pairs. These figures show how closely each calibration matches with the experiments and present the best and worst predictions of each calibration.

Figure A.6 shows the calibration with micropillars 1, 4 and 5, and. Figure A.7 shows the predictions of the same model for micropillars 2 and 3, which show very good agreement with the experiment. Finally, according to Table A.4 calibration to micropillars 1, 3 and 5 produced results similar to calibration with 1, 4 and 5, with a slightly smaller sum of absolute error.

The total sum of absolute error in calibrating the non-Schmid model with only one micropillar varies between $27 \%$ and $114 \%$ (Table A.2. The accuracy of the calibration highly depends on the orientation of the material used for the calibration. On the other hand, using two micropillars for calibration of the CPFE model produces maximum total error of about $28 \%$, regardless of which pair is used for calibration (Table A.4). Adding a third micropillar to the calibration only negligibly improves the results (Table A.4.

The same process was repeated for the Schmid modeling of QP980 ferrite micropillars. Tables A.5 and A.7 show the calibrated material parameters. According to Table A.6 the total prediction error when the Schmid model was calibrated with only one micropillar ranged from $58.4 \%$ to $109.29 \%$. Table A.8 
Figure A.1: Calibrating the non-Schmid model with QP980 ferrite micropillar 1. The circles show the force-displacement values measured in the micropillar compression test. The line shows the fitting of the model to the experiment.

shows that the range of sum of absolute error for calibrating with pairs of micropillars was improved $60.62 \%$ to $79.54 \%$, and for sets of three micropillars the errors were $63.46 \%$ and $67.17 \%$. It can be concluded that the accuracy of the Schmid model calibrated with sets of three micropillars did not improve as much as it did for the non-Schmid model. 


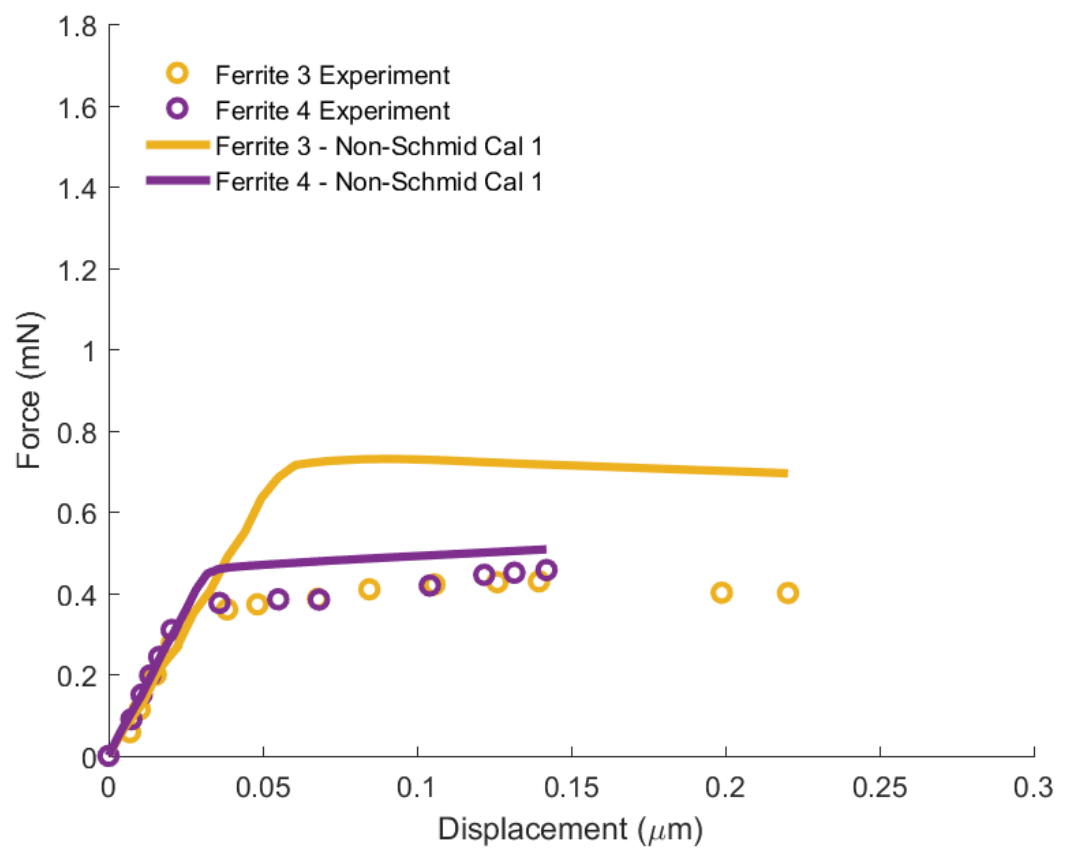

Figure A.2: The best and worst predictions of the non-Schmid model calibrated with QP980 micropillar 1. The circles show the force-displacement values measured in the experiments. The yellow and purple lines show the predictions of the model for deformation micropillar 3 and 4 respectively.

Table A.1: Material parameters found for the non-Schmid modeling of QP980 ferrite micropillar when the model was calibrated with one micropillar. In these calibrations $h_{0}=10$ and $\eta=3$.

\begin{tabular}{|c|r|r|r|r|r|r|}
\hline \multirow{2}{*}{$\begin{array}{c}\text { Material } \\
\text { Parameter }\end{array}$} & \multicolumn{6}{|c|}{ Calibration of the non-Schmid model to micropillar } \\
\cline { 2 - 7 } & 1 & \multicolumn{1}{c|}{2} & \multicolumn{1}{c|}{3} & \multicolumn{1}{c|}{4} & \multicolumn{1}{c|}{5} & \multicolumn{1}{c|}{6} \\
\hline$\tau_{s}$ & 562 & 716 & 1000 & 945 & 814 & 886 \\
\hline$\tau_{0}$ & 224 & 261 & 204 & 294 & 395 & 383 \\
\hline$a_{1}$ & 0.1931 & 0.4109 & 0.8736 & 0.1343 & 0.5092 & 0.0821 \\
\hline$a_{2}$ & 0.0062 & 0.5261 & 0.3319 & 0.9585 & 0.7091 & 0.4543 \\
\hline$a_{3}$ & 0.2432 & 0.4841 & 0.4157 & 0.0718 & 0.1441 & 0.9938 \\
\hline
\end{tabular}




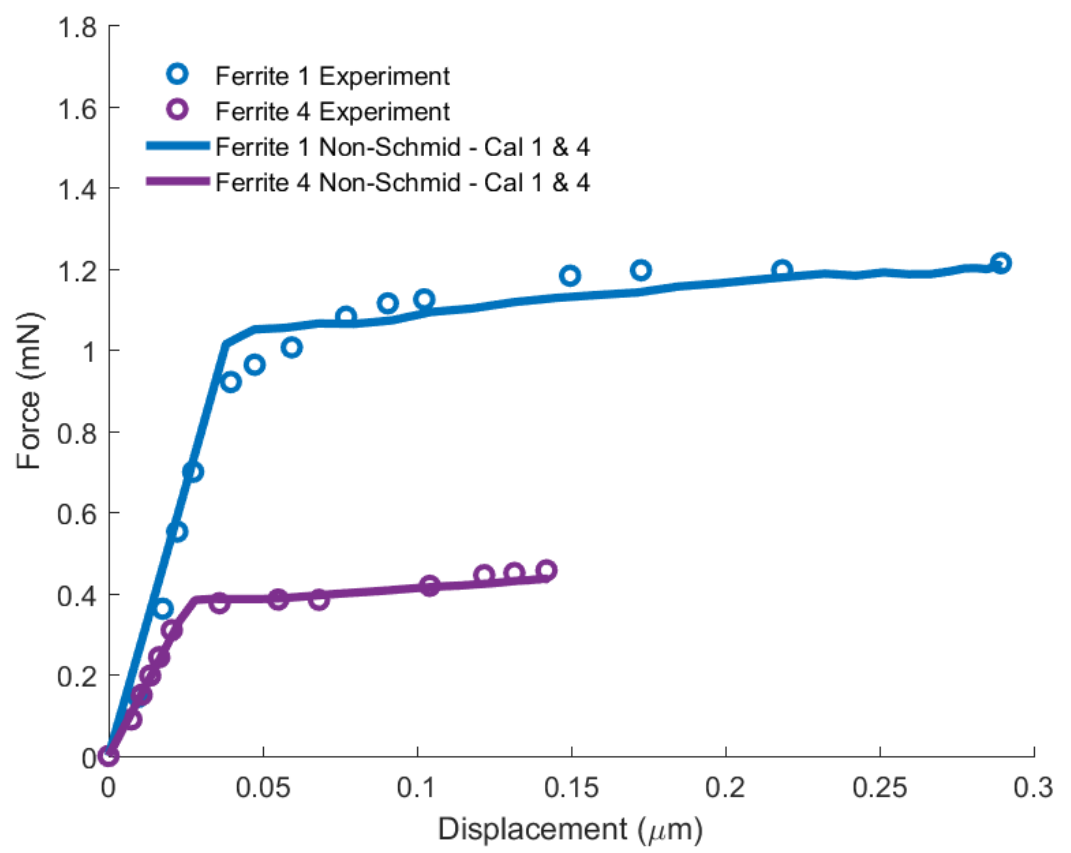

Figure A.3: Calibration of the non-Schmid model simultaneously to micropillars 1 and 4 . The circles show the force-displacement values measured in the experiments, and the solid lines represent the fitting of the models. 


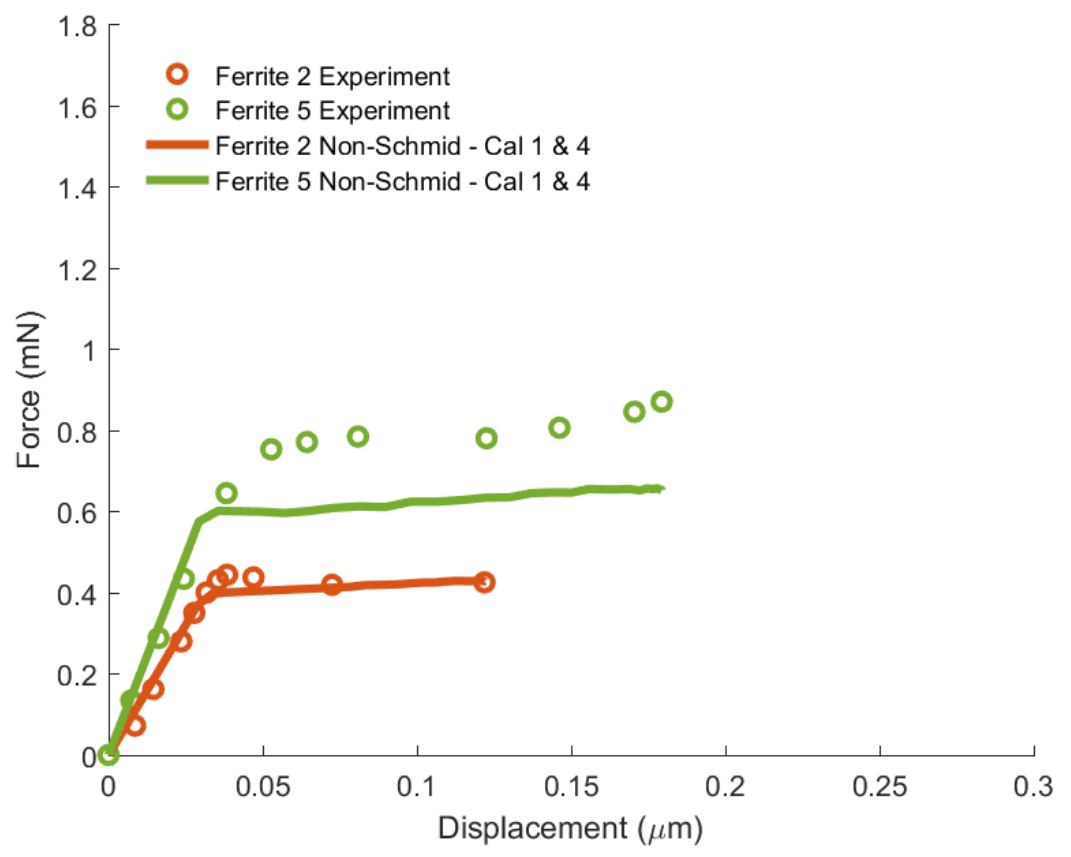

Figure A.4: The best and worst predictions of the non-Schimd model calibrated with micropillars 1 and 4 . The circles show the experimental measurements, and the solid lines show the force-displacement predictions of the model. 


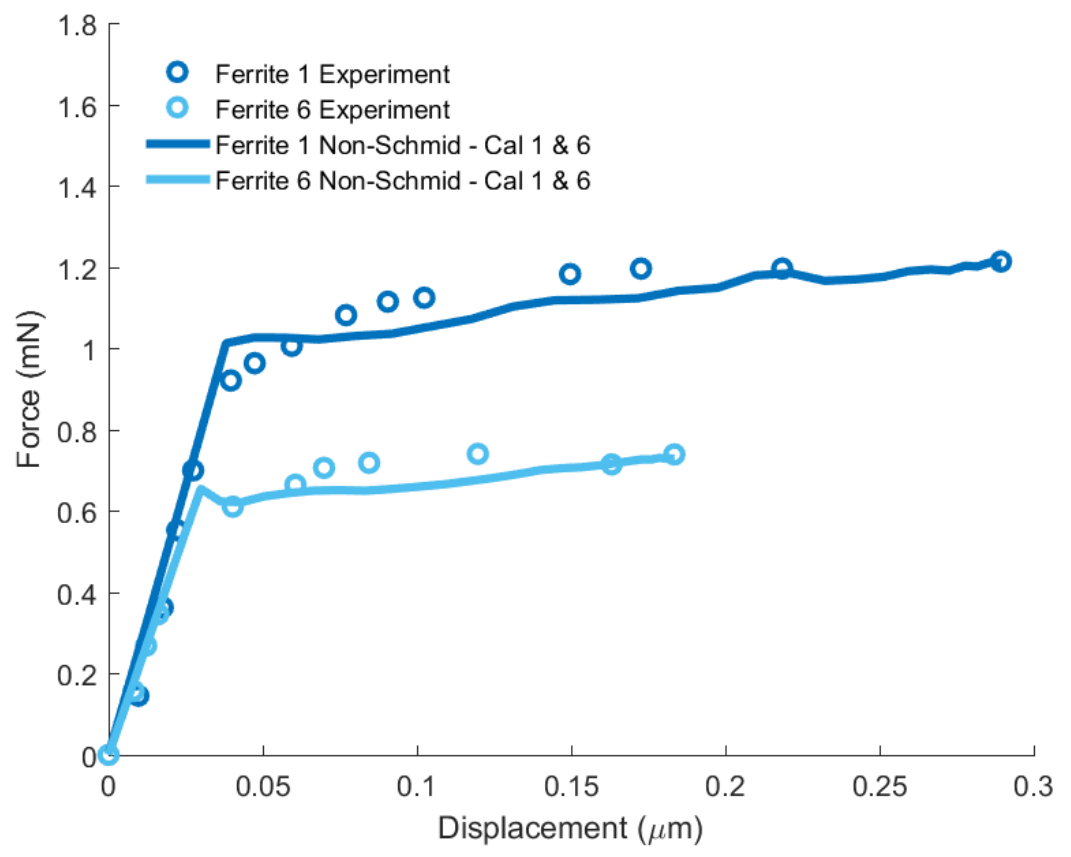

(a)

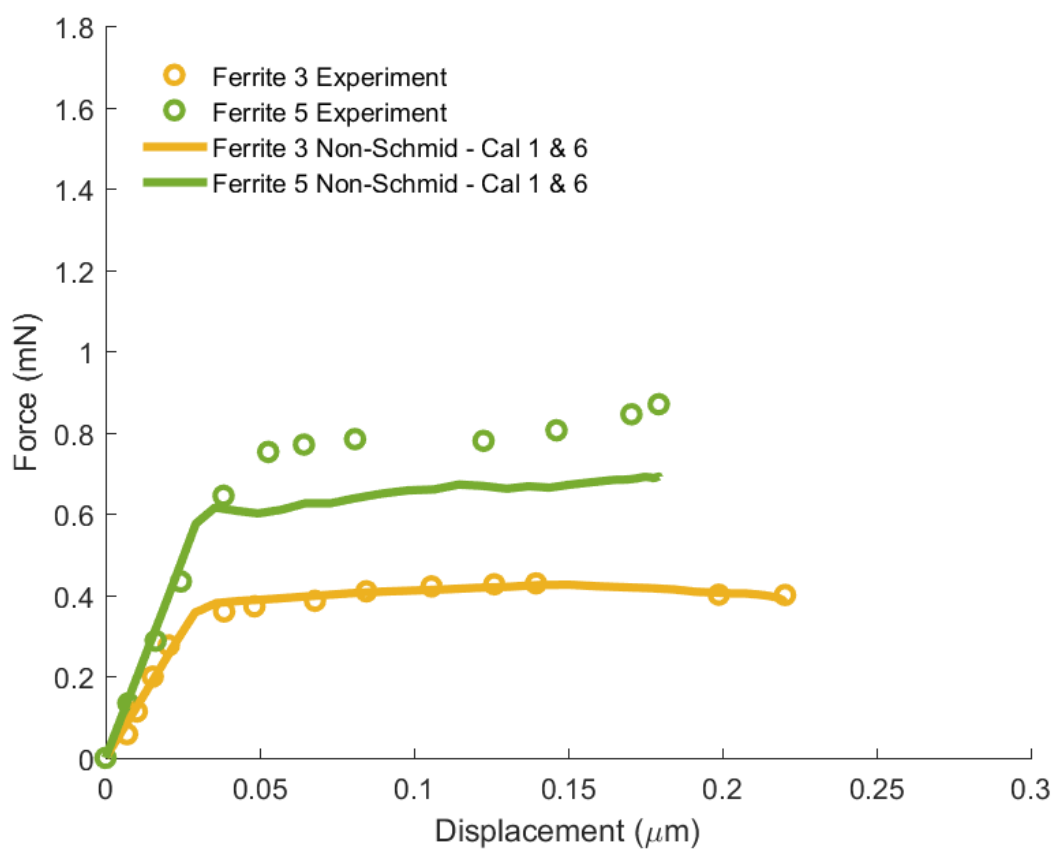

(b) 


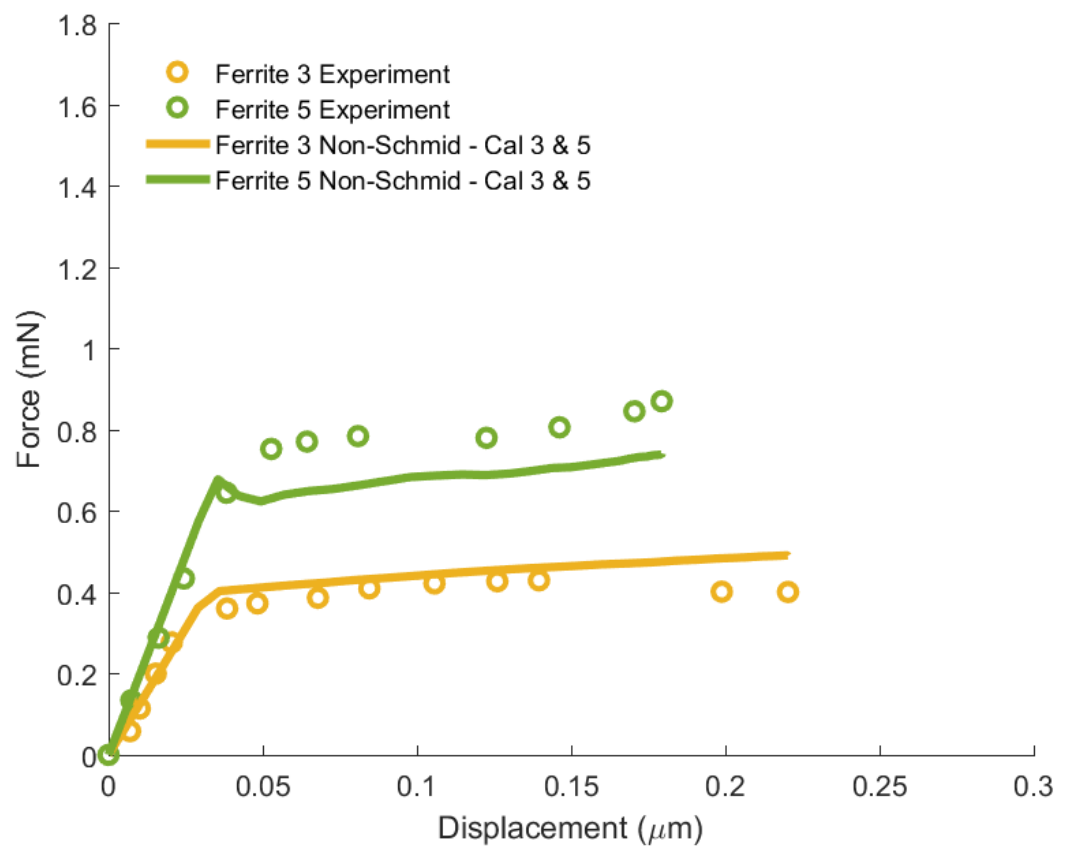

(c)

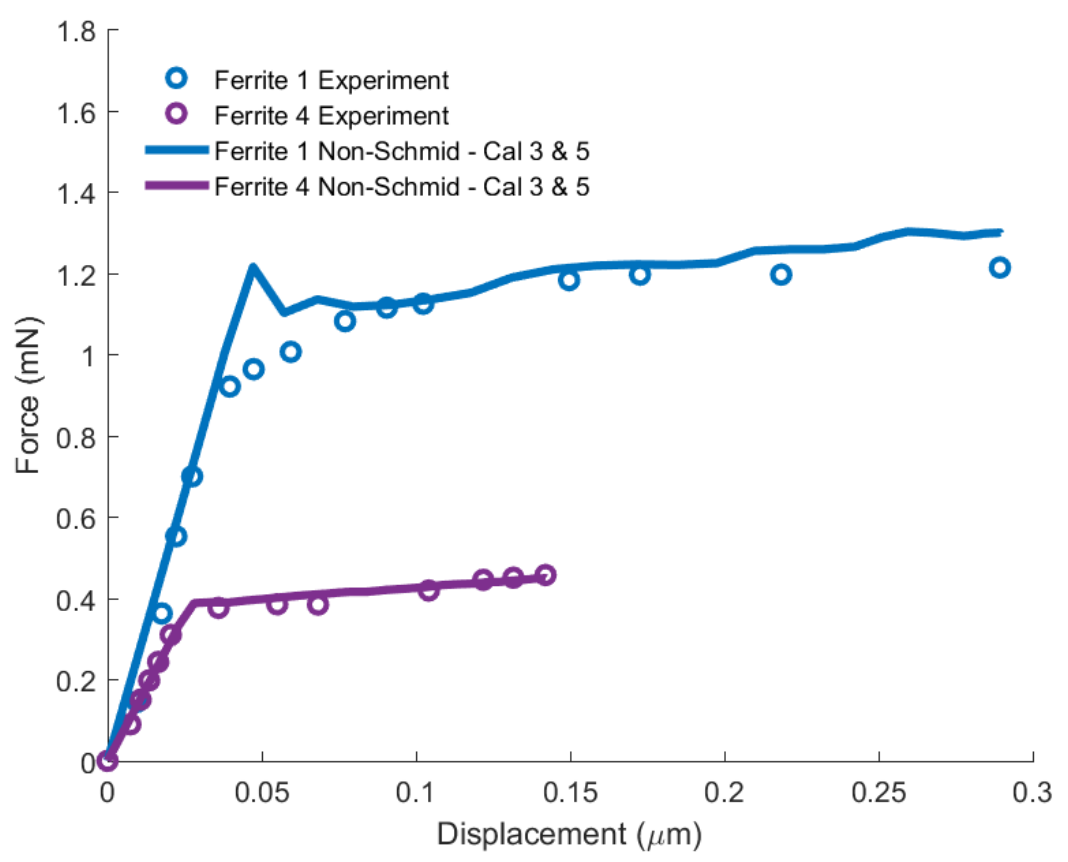

(d) 


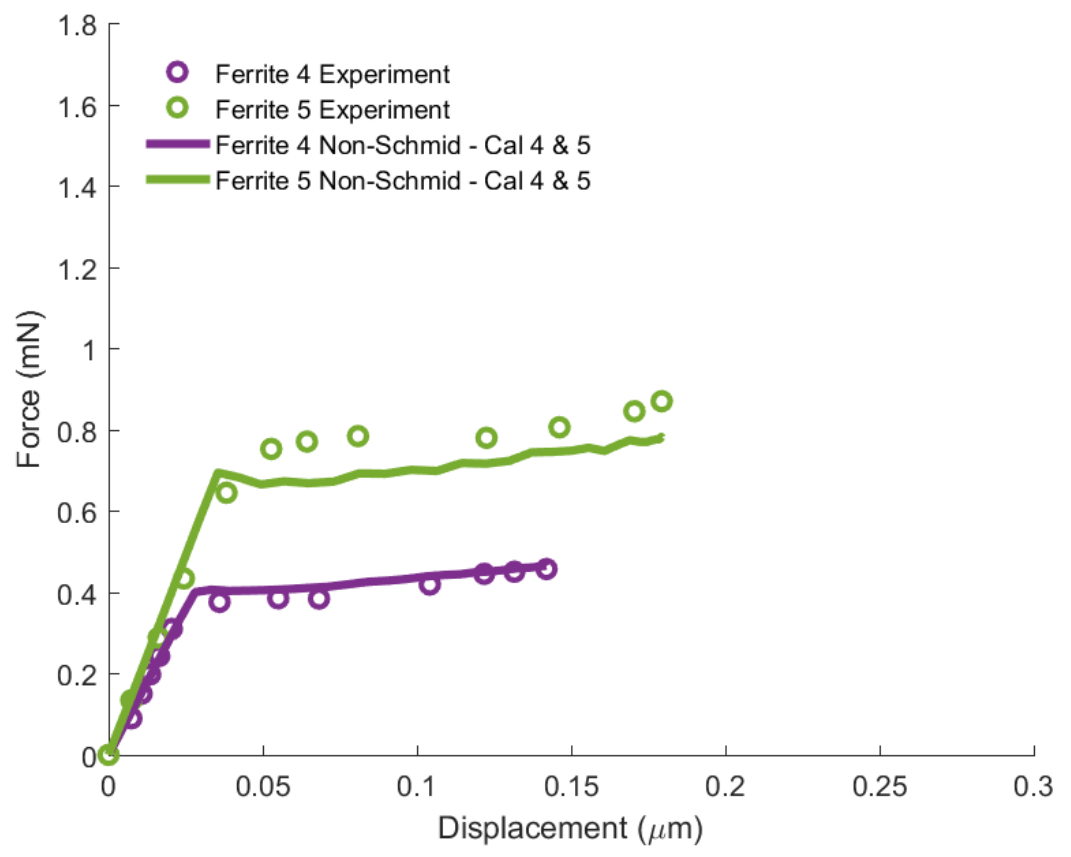

(e)

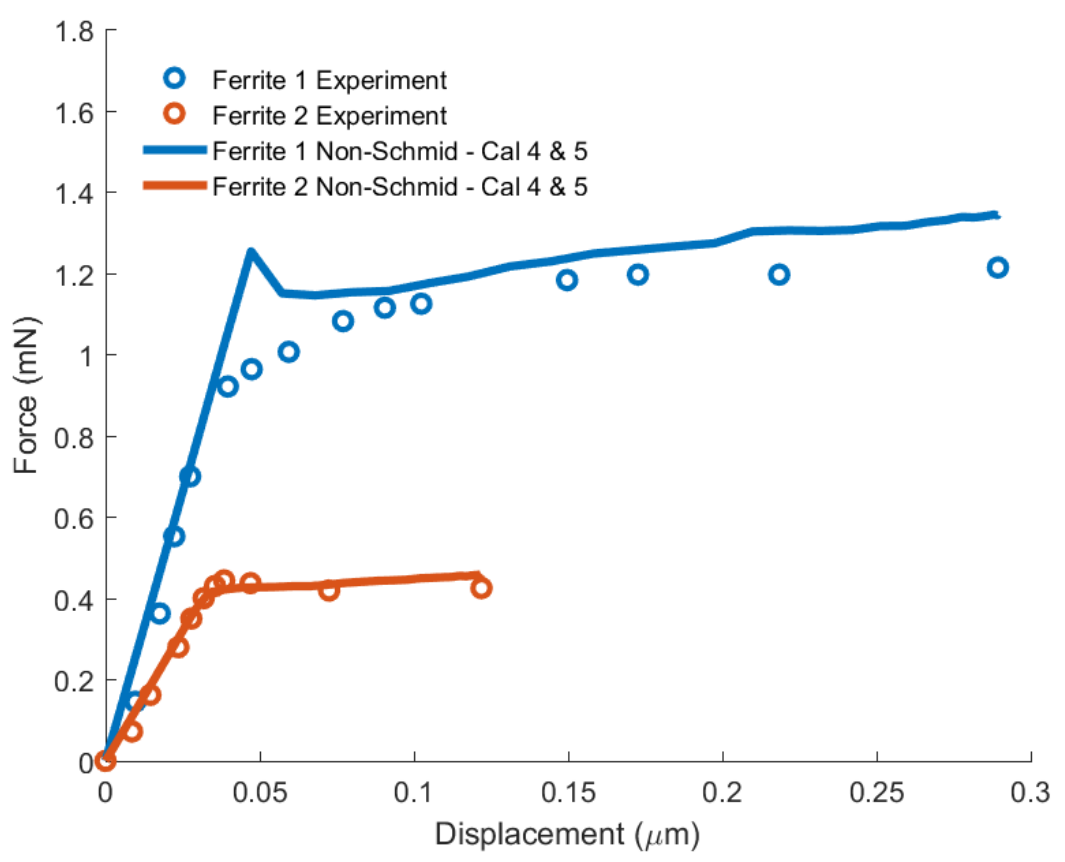

(f) 
Figure A.5: a) The non-Schmid model simultaneously calibrated to micropillars 1 and $6, \mathrm{~b}$ ) the best and worst predictions with this calibration. c) The non-Schmid model simultaneously calibrated to micropillar 2 and 5 , d) the best and worst predictions with this calibration. e) The non-Schmid model simultaneously calibrated to micropillar 4 and $5, \mathrm{f}$ ) the best and worst predictions with this calibration.

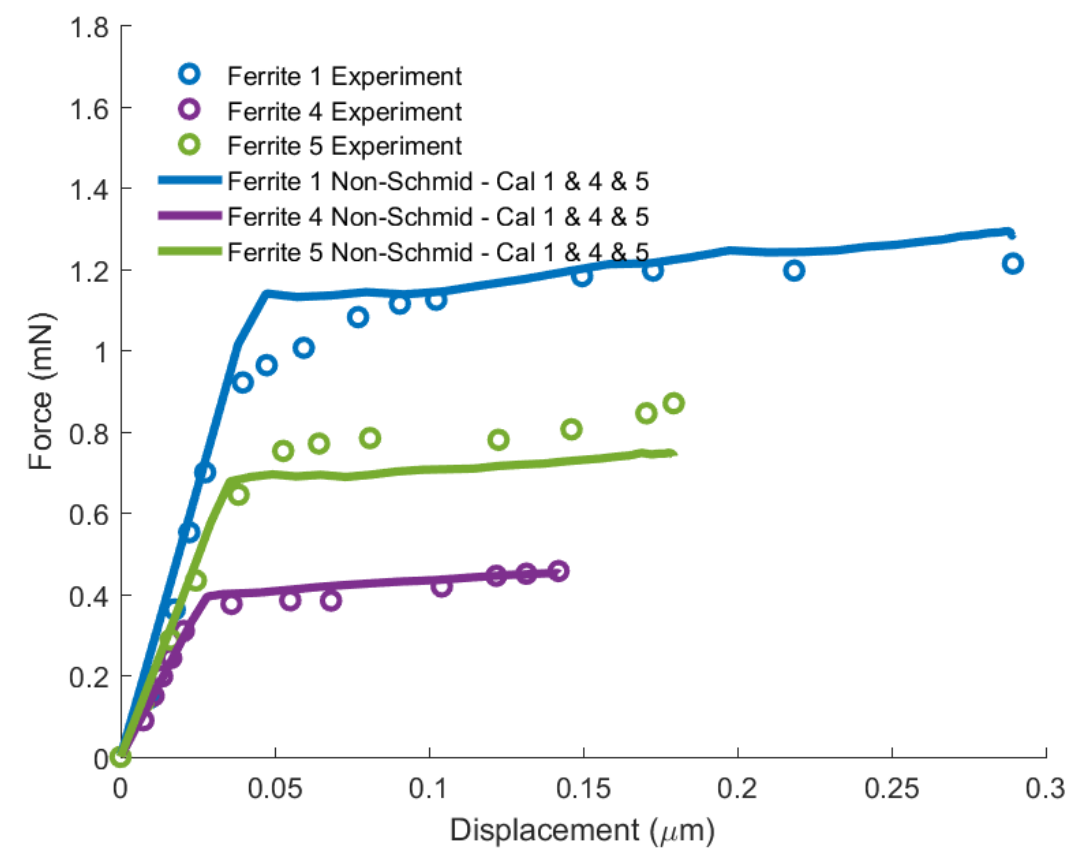

Figure A.6: Calibration of the non-Schmid model simultaneously to micropillars 1, 4 and 5. The circles shows the force-displacement values measured in the experiments and the solid lines represent the fitting of the models. 


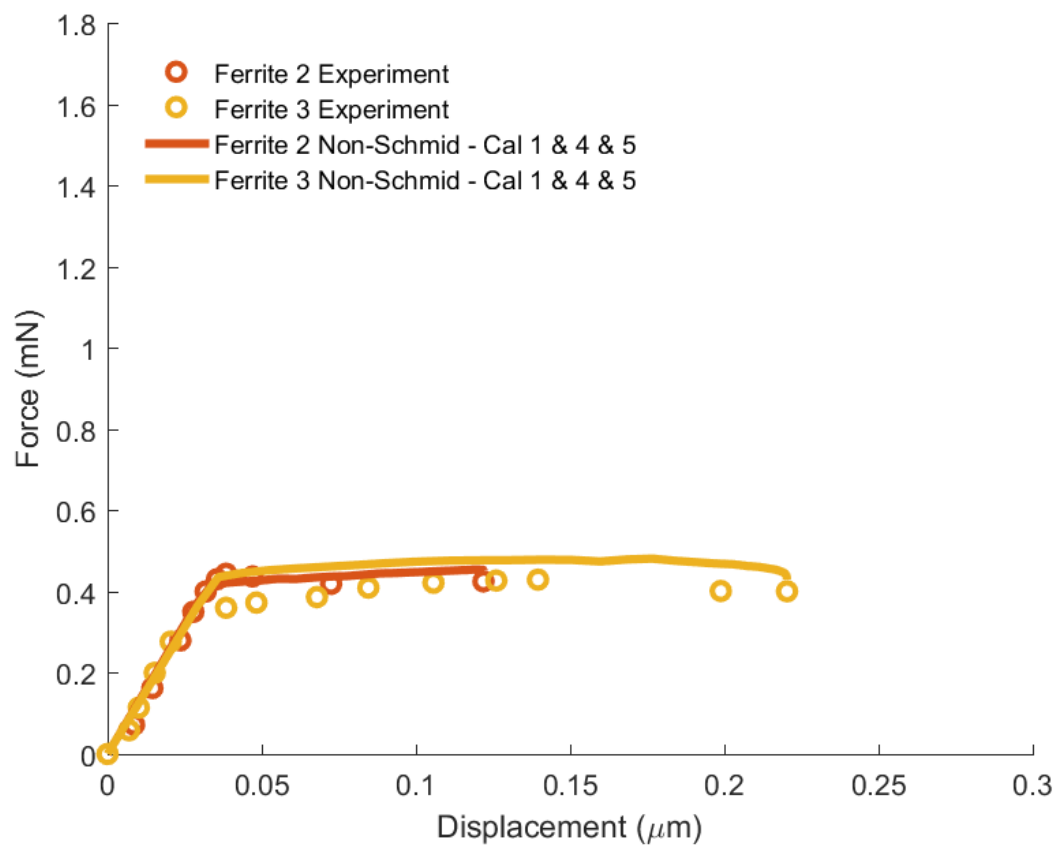

Figure A.7: The best and worst predictions of the non-Schimd model calibrated with micropillars 1, 4 and 5. The circles show the experimental measurements and the solid lines show the force-displacement predictions of the model. 
Table A.2: S-NRMSE for non-Schmid model calibrated to a single micropillar experiment at a time. A negative number represents underestimation and a positive number indicates overestimation of experimental data.

\begin{tabular}{|c|r|r|r|r|r|r|}
\hline \multirow{2}{*}{$\begin{array}{c}\text { Predicting } \\
\text { Micropillar }\end{array}$} & \multicolumn{6}{|c|}{ S-NRMSE for calibration of the non-Schmid model } \\
\cline { 2 - 7 } & \multicolumn{1}{c|}{1} & \multicolumn{1}{c|}{2} & \multicolumn{1}{c|}{3} & \multicolumn{1}{c|}{4} & \multicolumn{1}{c|}{5} & \multicolumn{1}{c|}{ to micropillar (\%) } \\
\hline 1 & 2.97 & -4.85 & -19.88 & 4.09 & 12.19 & 14.63 \\
\hline 2 & 8.50 & 3.02 & -16.84 & -4.13 & 8.79 & 18.09 \\
\hline 3 & 37.68 & 15.29 & -2.57 & 4.43 & 23.82 & 44.73 \\
\hline 4 & 8.36 & 2.43 & -17.13 & -2.07 & 10.95 & 27.59 \\
\hline 5 & -16.51 & -18.43 & -29.40 & -9.62 & 2.93 & -6.85 \\
\hline 6 & -13.31 & -14.48 & -27.68 & -2.61 & 8.00 & 2.10 \\
\hline Sum of absolute error & 87.33 & 58.50 & 113.49 & 26.95 & 66.68 & 114.00 \\
\hline
\end{tabular}

Table A.3: Material parameters found for the non-Schmid modeling of QP980 ferrite micropillar when the model was simultaneously calibrated with multiple micropillars. In these calibrations $h_{0}=10$ and $\eta=3$.

\begin{tabular}{|c|r|r|r|r|r|r|r|}
\hline \multirow{2}{*}{$\begin{array}{c}\text { Material } \\
\text { Parameters }\end{array}$} & \multicolumn{6}{|c|}{ Calibration of the non-Schmid model to micropillars } \\
\cline { 2 - 8 } & $1 \& 3$ & $1 \& 4$ & $1 \& 6$ & $3 \& 5$ & $4 \& 5$ & $1 \& 3 \& 5$ & $1 \& 4 \& 5$ \\
\hline$\tau_{s}$ & 570 & 760 & 880 & 400 & 555 & 537 & 445 \\
\hline$\tau_{0}$ & 257 & 280 & 276 & 300 & 306 & 249 & 269 \\
\hline$a_{1}$ & 0.0034 & 0.1454 & 0.0297 & 0 & 0.0045 & 0.0103 & 0.0618 \\
\hline$a_{2}$ & 0.8814 & 0.9204 & 0.9993 & 1 & 0.9646 & 0.5748 & 0.7022 \\
\hline$a_{3}$ & 0.3442 & 0.2546 & 0.1250 & 0 & 0.0127 & 0.0007 & 0.0084 \\
\hline
\end{tabular}


Table A.4: S-NRMSE for non-Schmid model simultaneously calibrated to multiple micropillar experiments at a time. A negative number indicates underestimation and a positive number represents overestimation of experimental data.

\begin{tabular}{|c|r|r|r|r|r|r|r|}
\hline \multirow{2}{*}{$\begin{array}{c}\text { Predicting } \\
\text { Micropillar }\end{array}$} & \multicolumn{7}{|c|}{ S-NRMSE for calibration of the non-Schmid model } \\
\cline { 2 - 9 } & $1 \& 3$ & $1 \& 4$ & $1 \& 6$ & $3 \& 5$ & $4 \& 5$ & $1 \& 3 \& 5$ & $1 \& 4 \& 5$ \\
\hline 1 & -4.18 & 3.52 & 3.74 & 5.36 & 6.58 & 3.80 & 4.74 \\
\hline 2 & -5.93 & -3.92 & -5.36 & -3.74 & 3.26 & -4.86 & 3.15 \\
\hline 3 & -2.56 & 4.50 & 2.52 & 6.78 & 6.09 & 2.79 & 8.30 \\
\hline 4 & -3.89 & -1.96 & -3.29 & 1.85 & 2.41 & -2.81 & 2.52 \\
\hline 5 & -11.09 & -10.97 & -9.30 & -7.28 & -5.35 & -8.98 & -5.67 \\
\hline 6 & -3.72 & -3.72 & -3.27 & 2.87 & 5.65 & -2.69 & 3.16 \\
\hline Sum of absolute error & 31.38 & 28.59 & 27.48 & 27.88 & 29.35 & 25.92 & 27.54 \\
\hline
\end{tabular}

Table A.5: Material parameters found from calibration of the Schmid model to single micropillars of QP980 ferrite. In these calibrations $h_{0} 1=h_{0} 2=h_{0} 3$ and $\eta_{1}=\eta_{2}=\eta_{3}=3$.

\begin{tabular}{|c|c|r|r|r|r|c|}
\hline \multirow{2}{*}{$\begin{array}{c}\text { Material } \\
\text { Parameters }\end{array}$} & \multicolumn{6}{|c|}{ Calibration of the Schmid model } \\
\cline { 2 - 7 } & 1 & \multicolumn{1}{|c|}{2} & \multicolumn{1}{|c|}{ to micropillar } \\
\hline$\tau_{s 1}$ & 615 & 400 & 881 & 400 & \multicolumn{1}{c|}{5} & 6 \\
\hline$\tau_{s 2}$ & 615 & 400 & 881 & 400 & 944 & 557 \\
\hline$\tau_{s 3}$ & 685 & 470 & 951 & 470 & 1014 & 627 \\
\hline$\tau_{01}$ & 185 & 191 & 113 & 183 & 260 & 238 \\
\hline$\tau_{02}$ & 195 & 201 & 123 & 193 & 270 & 248 \\
\hline$\tau_{03}$ & 305 & 311 & 233 & 303 & 380 & 358 \\
\hline$h_{01}$ & 272 & 10 & 66 & 10 & 98 & 66 \\
\hline
\end{tabular}


Table A.6: S-NRMSE for Schmid model calibrated to one micropillar experiment at a time. A negative number represents underestimation and a positive number indicates overestimation of experimental data.

\begin{tabular}{|c|r|r|r|r|r|c|}
\hline \multirow{2}{*}{$\begin{array}{c}\text { Predicting } \\
\text { Micropillar }\end{array}$} & \multicolumn{6}{|c|}{ S-NRMSE for calibration of the Schmid model } \\
& \multicolumn{1}{|c|}{1} & \multicolumn{1}{|c|}{2} & 3 & 4 & 5 & 6 \\
\hline 1 & 2.71 & -3.26 & -20.15 & -4.34 & 15.56 & 10.29 \\
\hline 2 & 4.52 & 3.80 & -16.83 & 3.17 & 15.62 & 11.76 \\
\hline 3 & 28.18 & 24.56 & 2.56 & 22.14 & 47.75 & 39.57 \\
\hline 4 & 2.46 & 2.54 & -16.28 & -2.36 & 17.37 & 12.17 \\
\hline 5 & -12.35 & -13.85 & -27.43 & -15.25 & 2.59 & -5.33 \\
\hline 6 & -9.35 & -10.39 & -26.04 & -11.97 & 5.66 & 2.13 \\
\hline Sum of absolute error & 59.56 & 58.40 & 109.29 & 59.22 & 104.56 & 81.26 \\
\hline
\end{tabular}

Table A.7: Material parameters found from calibration of the Schmid model to single micropillars of QP980 ferrite. In these calibrations $h_{0} 1=h_{0} 2=h_{0} 3$ and $\eta_{1}=\eta_{2}=\eta_{3}=3$.

\begin{tabular}{|c|r|r|r|r|r|r|r|}
\hline \multirow{2}{*}{$\begin{array}{c}\text { Material } \\
\text { Parameters }\end{array}$} & \multicolumn{6}{|c|}{ Calibration of the Schmid model to micropillars } \\
\cline { 2 - 8 } & $1 \& 3$ & $1 \& 4$ & $1 \& 6$ & $3 \& 5$ & $4 \& 5$ & $1 \& 3 \& 5$ & $1 \& 4 \& 5$ \\
\hline$\tau_{s 1}$ & 400 & 650 & 751 & 400 & 1000 & 400 & 1000 \\
\hline$\tau_{s 2}$ & 400 & 650 & 751 & 400 & 1000 & 400 & 1000 \\
\hline$\tau_{s 3}$ & 470 & 720 & 821 & 470 & 1070 & 470 & 1070 \\
\hline$\tau_{01}$ & 150 & 195 & 207 & 159 & 200 & 173 & 185 \\
\hline$\tau_{02}$ & 160 & 205 & 217 & 169 & 210 & 183 & 195 \\
\hline$\tau_{03}$ & 270 & 330 & 327 & 279 & 320 & 293 & 330 \\
\hline$h_{01}$ & 10 & 110 & 154 & 10 & 400 & 10 & 400 \\
\hline
\end{tabular}


Table A.8: S-NRMSE for Schmid model simultaneously calibrated to multiple micropillar experiments at a time. A negative number indicates underestimation and a positive number represents overestimation of experimental data.

\begin{tabular}{|c|r|r|r|r|r|r|r|}
\hline \multirow{2}{*}{$\begin{array}{c}\text { Predicting } \\
\text { Micropillar }\end{array}$} & \multicolumn{6}{|c|}{ S-NRMSE for calibration of the Schmid model to micropillars (\%) } \\
\cline { 2 - 8 } & $1 \& 3$ & $1 \& 4$ & $1 \& 6$ & $3 \& 5$ & $4 \& 5$ & $1 \& 3 \& 5$ & $1 \& 4 \& 5$ \\
\hline 1 & -11.70 & 3.23 & 5.34 & -9.49 & 6.84 & -6.36 & 4.47 \\
\hline 2 & -7.91 & 5.18 & 7.56 & -5.87 & 8.42 & 3.68 & 6.56 \\
\hline 3 & 10.82 & 27.93 & 33.01 & 13.91 & 38.35 & 18.37 & 34.53 \\
\hline 4 & -8.55 & 3.84 & 6.81 & -6.47 & 7.85 & -3.70 & 4.91 \\
\hline 5 & -21.49 & -11.93 & -9.11 & -19.73 & -7.41 & -17.18 & -9.75 \\
\hline 6 & -19.07 & -8.51 & -5.54 & -17.08 & -4.56 & -14.17 & -6.95 \\
\hline Sum of absolute error & 79.54 & 60.62 & 67.36 & 72.55 & 73.42 & 63.46 & 67.17 \\
\hline
\end{tabular}

